\title{
On Modelling of Mass Transport for Formulation of Conditions for Increasing Integration Rate of Elements Framework Current Comparator. an Approach to Optimize of Technological Process
}

\author{
E.L. Pankratov \\ Nizhny Novgorod State University, 23 Gagarin avenue, Nizhny Novgorod, 603950, Russia \\ Nizhny Novgorod State Technical University, 24 Minin Street, Nizhny Novgorod, 603950, Russia
}

\begin{abstract}
*Corresponding Author: E.L. Pankratov, Nizhny Novgorod State University, 23 Gagarin avenue, Nizhny Novgorod, 603950, Russia; Nizhny Novgorod State Technical University, 24 Minin Street, Nizhny Novgorod, 603950, Russia
\end{abstract}

\begin{abstract}
In this paper we introduce an analytical approach to analyze mass and heat transport in heterostructures during manufacturing of integrated circuits with account nonlinearity of processes. The approach gives a possibility to analyze mass and heat transport in multilayer structures without crosslinking of solutions on interfaces between layers. The approach also gives a possibility to take into account spatial and temporal variation of parameters of considered processes. Based on this approach we analyzed manufacturing a current comparator to increase density of elements.
\end{abstract}

Keywords: current comparator; optimization of manufacturing; analytical approach for modelling.

\section{INTRODUCTION}

An actual and intensively solving problems of solid state electronics is increasing of integration rate of elements of integrated circuits ( $p$ - $n$-junctions, their systems et al) [1-8]. Increasing of the integration rate leads to necessity to decrease their dimensions. To decrease the dimensions are using several approaches. They are widely using laser and microwave types of annealing of infused dopants. These types of annealing are also widely using for annealing of radiation defects, generated during ion implantation [9-17]. Using the approaches gives a possibility to increase integration rate of elements of integrated circuits through inhomogeneity of technological parameters due to generating inhomogenous distribution of temperature. In this situation one can obtain decreasing dimensions of elements of integrated circuits [18] with account Arrhenius law [1,3]. Another approach to manufacture elements of integrated circuits with smaller dimensions is doping of heterostructure by diffusion or ion implantation [1-3]. However in this case optimization of dopant and/or radiation defects is required [18].

In this paper we consider a heterostructure. The heterostructure consist of a substrate and several epitaxial layers. Some sections have been manufactured in the epitaxial layers. Further we consider doping of these sections by diffusion or ion implantation. The doping gives a possibility to manufacture field-effect transistors framework a cascaded-inverter circuit so as it is shown on Figs. 1. The manufacturing gives a possibility to increase density of elements of the considered in [4] current comparator. After the considered doping dopant and/or radiation defects should be annealed. Framework the paper we analyzed dynamics of redistribution of dopant and/or radiation defects during their annealing. We introduce an approach to decrease dimensions of the element. However it is necessary to complicate technological process. 
On Modelling of Mass Transport for Formulation of Conditions for Increasing Integration Rate of Elements Framework Current Comparator. an Approach to Optimize of Technological Process

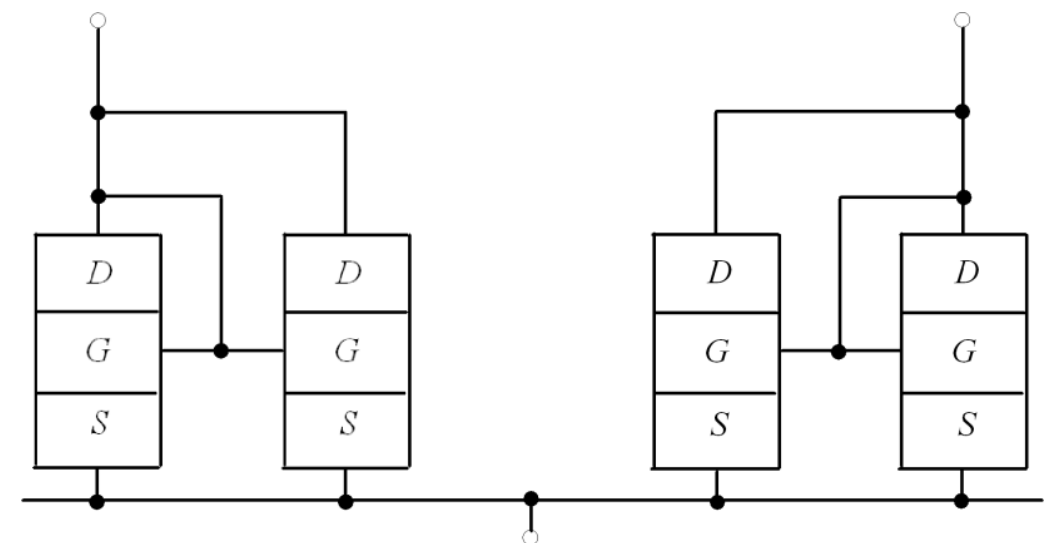

Fig1. The considered current comparator [4]

\section{Method OF SOlution}

In this section we determine spatio-temporal distributions of concentrations of infused and implanted dopants. To determine these distributions we calculate appropriate solutions of the second Fick's law $[1,3,18]$

$$
\begin{aligned}
& \frac{\partial C(x, y, z, t)}{\partial t}= \\
& =\frac{\partial}{\partial x}\left[D_{C} \frac{\partial C(x, y, z, t)}{\partial x}\right]+\frac{\partial}{\partial y}\left[D_{C} \frac{\partial C(x, y, z, t)}{\partial y}\right]+\frac{\partial}{\partial z}\left[D_{C} \frac{\partial C(x, y, z, t)}{\partial z}\right] .
\end{aligned}
$$

Boundary and initial conditions for the equations are

$$
\begin{aligned}
& \left.\frac{\partial C(x, y, z, t)}{\partial x}\right|_{x=0}=0,\left.\frac{\partial C(x, y, z, t)}{\partial x}\right|_{x=L_{x}}=0,\left.\frac{\partial C(x, y, z, t)}{\partial y}\right|_{y=0}=0,\left.\frac{\partial C(x, y, z, t)}{\partial y}\right|_{x=L_{y}}=0, \\
& \left.\frac{\partial C(x, y, z, t)}{\partial z}\right|_{z=0}=0,\left.\frac{\partial C(x, y, z, t)}{\partial z}\right|_{x=L_{z}}=0, C(x, y, z, 0)=f(x, y, z) .
\end{aligned}
$$

The function $C(x, y, z, t)$ describes the spatio-temporal distribution of concentration of dopant; $T$ is the temperature of annealing; $D_{C}$ is the dopant diffusion coefficient. Value of dopant diffusion coefficient could be changed with changing materials of heterostructure, with changing temperature of materials (including annealing), with changing concentrations of dopant and radiation defects. We approximate dependences of dopant diffusion coefficient on parameters by the following relation with account results in Refs. [20-22]

$$
D_{C}=D_{L}(x, y, z, T)\left[1+\xi \frac{C^{\gamma}(x, y, z, t)}{P^{\gamma}(x, y, z, T)}\right]\left[1+\varsigma_{1} \frac{V(x, y, z, t)}{V^{*}}+\varsigma_{2} \frac{V^{2}(x, y, z, t)}{\left(V^{*}\right)^{2}}\right] \text {. }
$$

Here the function $D_{L}(x, y, z, T)$ describes the spatial (in heterostructure) and temperature (due to Arrhenius law) dependences of diffusion coefficient of dopant. The function $P(x, y, z, T)$ describes the limit of solubility of dopant. Parameter $\gamma \in[1,3]$ describes average quantity of charged defects interacted with atom of dopant [20]. The function $V(x, y, z, t)$ describes the spatio-temporal distribution of concentration of radiation vacancies. Parameter $V^{*}$ describes the equilibrium distribution of concentration of 
On Modelling of Mass Transport for Formulation of Conditions for Increasing Integration Rate of Elements Framework Current Comparator. an Approach to Optimize of Technological Process

vacancies. The considered concentrational dependence of dopant diffusion coefficient has been described in details in [20]. It should be noted, that using diffusion type of doping did not generation radiation defects. In this situation $\zeta_{1}=\zeta_{2}=0$. We determine spatio-temporal distributions of concentrations of radiation defects by solving the following system of equations [21,22]

$$
\begin{aligned}
& \frac{\partial I(x, y, z, t)}{\partial t}=\frac{\partial}{\partial x}\left[D_{I}(x, y, z, T) \frac{\partial I(x, y, z, t)}{\partial x}\right]+\frac{\partial}{\partial y}\left[D_{I}(x, y, z, T) \frac{\partial I(x, y, z, t)}{\partial y}\right]+ \\
& +\frac{\partial}{\partial z}\left[D_{I}(x, y, z, T) \frac{\partial I(x, y, z, t)}{\partial z}\right]-k_{I, V}(x, y, z, T) I(x, y, z, t) V(x, y, z, t)--k_{I, I}(x, y, z, T) I^{2}(x, y, z, t) \\
& \frac{\partial V(x, y, z, t)}{\partial t}=\frac{\partial}{\partial x}\left[D_{V}(x, y, z, T) \frac{\partial V(x, y, z, t)}{\partial x}\right]+\frac{\partial}{\partial y}\left[D_{V}(x, y, z, T) \frac{\partial V(x, y, z, t)}{\partial y}\right]+ \\
& +\frac{\partial}{\partial z}\left[D_{V}(x, y, z, T) \frac{\partial V(x, y, z, t)}{\partial z}\right]-k_{I, V}(x, y, z, T) I(x, y, z, t) V(x, y, z, t)+ \\
& +k_{V, V}(x, y, z, T) V^{2}(x, y, z, t) .
\end{aligned}
$$

Boundary and initial conditions for these equations are

$$
\begin{aligned}
& \left.\frac{\partial \rho(x, y, z, t)}{\partial x}\right|_{x=0}=0,\left.\frac{\partial \rho(x, y, z, t)}{\partial x}\right|_{x=L_{x}}=0,\left.\frac{\partial \rho(x, y, z, t)}{\partial y}\right|_{y=0}=0,\left.\frac{\partial \rho(x, y, z, t)}{\partial y}\right|_{y=L_{y}}=0, \\
& \left.\frac{\partial \rho(x, y, z, t)}{\partial z}\right|_{z=0}=0,\left.\frac{\partial \rho(x, y, z, t)}{\partial z}\right|_{z=L_{z}}=0, \rho(x, y, z, 0)=f_{\rho}(x, y, z) .
\end{aligned}
$$

Here $\rho=I, V$. The function $I(x, y, z, t)$ describes the spatio-temporal distribution of concentration of radiation interstitials; $D_{\rho}(x, y, z, T)$ are the diffusion coefficients of point radiation defects; terms $V^{2}(x, y, z, t)$ and $I^{2}(x, y, z, t)$ correspond to generation divacancies and diinterstitials; $k_{I, V}(x, y, z, T)$ is the parameter of recombination of point radiation defects; $k_{I, I}(x, y, z, T)$ and $k_{V, V}(x, y, z, T)$ are the parameters of generation of simplest complexes of point radiation defects.

Further we determine distributions in space and time of concentrations of divacancies $\Phi_{V}(x, y, z, t)$ and diinterstitials $\Phi_{I}(x, y, z, t)$ by solving the following system of equations [21,22]

$$
\begin{aligned}
& \frac{\partial \Phi_{I}(x, y, z, t)}{\partial t}=\frac{\partial}{\partial x}\left[D_{\Phi I}(x, y, z, T) \frac{\partial \Phi_{I}(x, y, z, t)}{\partial x}\right]+\frac{\partial}{\partial y}\left[D_{\Phi I}(x, y, z, T) \frac{\partial \Phi_{I}(x, y, z, t)}{\partial y}\right]+ \\
& +\frac{\partial}{\partial z}\left[D_{\Phi I}(x, y, z, T) \frac{\partial \Phi_{I}(x, y, z, t)}{\partial z}\right]+k_{I, I}(x, y, z, T) I^{2}(x, y, z, t)-k_{I}(x, y, z, T) I(x, y, z, t) \\
& \frac{\partial \Phi_{V}(x, y, z, t)}{\partial t}=\frac{\partial}{\partial x}\left[D_{\Phi V}(x, y, z, T) \frac{\partial \Phi_{V}(x, y, z, t)}{\partial x}\right]+\frac{\partial}{\partial y}\left[D_{\Phi V}(x, y, z, T) \frac{\partial \Phi_{V}(x, y, z, t)}{\partial y}\right]+ \\
& +\frac{\partial}{\partial z}\left[D_{\Phi V}(x, y, z, T) \frac{\partial \Phi_{V}(x, y, z, t)}{\partial z}\right]+k_{V, V}(x, y, z, T) V^{2}(x, y, z, t)-k_{V}(x, y, z, T) V(x, y, z, t) .
\end{aligned}
$$


On Modelling of Mass Transport for Formulation of Conditions for Increasing Integration Rate of Elements Framework Current Comparator. an Approach to Optimize of Technological Process

Boundary and initial conditions for these equations are

$$
\begin{aligned}
\left.\frac{\partial \Phi_{\rho}(x, y, z, t)}{\partial x}\right|_{x=0}=0,\left.\frac{\partial \Phi_{\rho}(x, y, z, t)}{\partial x}\right|_{x=L_{x}}=0,\left.\frac{\partial \Phi_{\rho}(x, y, z, t)}{\partial y}\right|_{y=0}=0,\left.\frac{\partial \Phi_{\rho}(x, y, z, t)}{\partial y}\right|_{y=L_{y}}=0, \\
\left.\frac{\partial \Phi_{\rho}(x, y, z, t)}{\partial z}\right|_{z=0}=0,\left.\frac{\partial \Phi_{\rho}(x, y, z, t)}{\partial z}\right|_{z=L_{z}}=0,
\end{aligned}
$$

$\Phi_{I}(x, y, z, 0)=f_{\Phi I}(x, y, z), \Phi_{V}(x, y, z, 0)=f_{\Phi V}(x, y, z)$.

Here $D_{\Phi \rho}(x, y, z, T)$ are the diffusion coefficients of the above complexes of radiation defects; $k_{I}(x, y, z, T)$ and $k_{V}(x, y, z, T)$ are the parameters of decay of these complexes.

We calculate distributions of concentrations of point radiation defects in space and time by recently elaborated approach [18]. The approach based on transformation of approximations of diffusion coefficients in the following form: $D_{\rho}(x, y, z, T)=D_{0 \rho}\left[1+\varepsilon_{\rho} g_{\rho}(x, y, z, T)\right]$, where $D_{0 \rho}$ are the average values of diffusion coefficients, $0 \leq \varepsilon_{\rho}<1,\left|g_{\rho}(x, y, z, T)\right| \leq 1, \rho=I, V$. We also used analogous transformation of approximations of parameters of recombination of point defects and parameters of generation of their complexes: $\quad k_{I, V}(x, y, z, T)=k_{0 I, V}\left[1+\varepsilon_{I, V} \quad g_{I, V}(x, y, z, T)\right], \quad k_{I, I}(x, y, z, T)=k_{0 I I I} \quad\left[1+\varepsilon_{I, I} \quad g_{I, I}(x, y, z, T)\right] \quad$ and $k_{V, V}(x, y, z, T)=k_{0 V, V}\left[1+\varepsilon_{V, V} g_{V, V}(x, y, z, T)\right]$, where $k_{0 \rho 1, \rho 2}$ are the their average values, $0 \leq \varepsilon_{I, V}<1,0 \leq \varepsilon_{I, I}<1$, $0 \leq \varepsilon_{V, V}<1,\left|g_{I, V}(x, y, z, T)\right| \leq 1,\left|g_{I, I}(x, y, z, T)\right| \leq 1,\left|g_{V, V}(x, y, z, T)\right| \leq 1$. Let us introduce the following dimensionless $\quad$ variables: $\quad \tilde{I}(x, y, z, t)=I(x, y, z, t) / I^{*}, \quad \tilde{V}(x, y, z, t)=\quad=V(x, y, z, t) / V^{*}$, $\omega=L^{2} k_{0 I, V} / \sqrt{D_{0 I} D_{0 V}}, \Omega_{\rho}=L^{2} k_{0 \rho, \rho} / \sqrt{D_{0 I} D_{0 V}}, \vartheta=\sqrt{D_{0 I} D_{0 V}} t / L^{2}, \chi=x / L_{x}, \eta=y / L_{y}, \phi=z / L_{z}$. The introduction leads to transformation of Eqs.(4) and conditions (5) to the following form

$$
\begin{aligned}
& \frac{\partial \tilde{I}(\chi, \eta, \phi, \vartheta)}{\partial \vartheta}=\frac{D_{0 I}}{\sqrt{D_{0 I} D_{0 V}}} \frac{\partial}{\partial \chi}\left\{\left[1+\varepsilon_{I} g_{I}(\chi, \eta, \phi, T)\right] \frac{\partial \tilde{I}(\chi, \eta, \phi, \vartheta)}{\partial \chi}\right\}+\frac{\partial}{\partial \eta}\left\{1+\varepsilon_{I} g_{I}(\chi, \eta, \phi, T)\right] \times \\
& \left.\times \frac{\partial \tilde{I}(\chi, \eta, \phi, \vartheta)}{\partial \eta}\right\} \frac{D_{0 I}}{\sqrt{D_{0 I} D_{0 V}}}+\frac{D_{0 I}}{\sqrt{D_{0 I} D_{0 V}}} \frac{\partial}{\partial \phi}\left\{\left[1+\varepsilon_{I} g_{I}(\chi, \eta, \phi, T)\right] \frac{\partial \tilde{I}(\chi, \eta, \phi, \vartheta)}{\partial \phi}\right\}-\tilde{I}(\chi, \eta, \phi, \vartheta) \times \\
& \times \omega\left[1+\varepsilon_{I, V} g_{I, V}(\chi, \eta, \phi, T)\right] \tilde{V}(\chi, \eta, \phi, \vartheta)-\Omega_{I}\left[1+\varepsilon_{I, I} g_{I, I}(\chi, \eta, \phi, T)\right] \tilde{I}^{2}(\chi, \eta, \phi, \vartheta) \\
& \frac{\partial \tilde{V}(\chi, \eta, \phi, \vartheta)}{\partial \vartheta}=\frac{D_{0 V}}{\sqrt{D_{0 I} D_{0 V}}} \frac{\partial}{\partial \chi}\left\{\left[1+\varepsilon_{V} g_{V}(\chi, \eta, \phi, T)\right] \frac{\partial \tilde{V}(\chi, \eta, \phi, \vartheta)}{\partial \chi}\right\}+\frac{\partial}{\partial \eta}\left\{1+\varepsilon_{V} g_{V}(\chi, \eta, \phi, T)\right] \times \\
& \left.\times \frac{\partial \tilde{V}(\chi, \eta, \phi, \vartheta)}{\partial \eta}\right\} \frac{D_{0 V}}{\sqrt{D_{0 I} D_{0 V}}}+\frac{D_{0 V}}{\sqrt{D_{0 I} D_{0 V}}} \frac{\partial}{\partial \phi}\left\{\left[1+\varepsilon_{V} g_{V}(\chi, \eta, \phi, T)\right] \frac{\partial \tilde{V}(\chi, \eta, \phi, \vartheta)}{\partial \phi}\right\}-\tilde{I}(\chi, \eta, \phi, \vartheta) \times \\
& \times \omega\left[1+\varepsilon_{I, V} g_{I, V}(\chi, \eta, \phi, T)\right] \tilde{V}(\chi, \eta, \phi, \vartheta)-\Omega_{V}\left[1+\varepsilon_{V, V} g_{V, V}(\chi, \eta, \phi, T)\right] \tilde{V}^{2}(\chi, \eta, \phi, \vartheta) \\
& \left.\frac{\partial \tilde{\rho}(\chi, \eta, \phi, \vartheta)}{\partial \chi}\right|_{\chi=0}=0,\left.\frac{\partial \tilde{\rho}(\chi, \eta, \phi, \vartheta)}{\partial \chi}\right|_{\chi=1}=0,\left.\frac{\partial \tilde{\rho}(\chi, \eta, \phi, \vartheta)}{\partial \eta}\right|_{\eta=0}=0,\left.\frac{\partial \tilde{\rho}(\chi, \eta, \phi, \vartheta)}{\partial \eta}\right|_{\eta=1}=0
\end{aligned}
$$


On Modelling of Mass Transport for Formulation of Conditions for Increasing Integration Rate of Elements Framework Current Comparator. an Approach to Optimize of Technological Process

$$
\left.\frac{\partial \tilde{\rho}(\chi, \eta, \phi, \vartheta)}{\partial \phi}\right|_{\phi=0}=0,\left.\frac{\partial \tilde{\rho}(\chi, \eta, \phi, \vartheta)}{\partial \phi}\right|_{\phi=1}=0, \tilde{\rho}(\chi, \eta, \phi, \vartheta)=\frac{f_{\rho}(\chi, \eta, \phi, \vartheta)}{\rho^{*}} .
$$

We determine solutions of Eqs.(8) with conditions (9) framework recently introduced approach [18], i.e. as the power series

$$
\tilde{\rho}(\chi, \eta, \phi, \vartheta)=\sum_{i=0}^{\infty} \varepsilon_{\rho}^{i} \sum_{j=0}^{\infty} \omega^{j} \sum_{k=0}^{\infty} \Omega_{\rho}^{k} \tilde{\rho}_{i j k}(\chi, \eta, \phi, \vartheta) .
$$

Substitution of the series (10) into Eqs.(8) and conditions (9) gives us possibility to obtain equations for initial-order approximations of concentration of point defects $\tilde{I}_{000}(\chi, \eta, \phi, \vartheta)$ and $\tilde{V}_{000}(\chi, \eta, \phi, \vartheta)$ and corrections for them $\tilde{I}_{i j k}(\chi, \eta, \phi, \vartheta)$ and $\tilde{V}_{i j k}(\chi, \eta, \phi, \vartheta), i \geq 1, j \geq 1, k \geq 1$. The equations are presented in the Appendix. Solutions of the equations could be obtained by standard Fourier approach $[24,25]$. The solutions are presented in the Appendix.

Now we calculate distributions of concentrations of simplest complexes of point radiation defects in space and time. To determine the distributions we transform approximations of diffusion coefficients in the following form: $D_{\Phi_{\rho}}(x, y, z, T)=D_{0 \varphi_{\rho}}\left[1+\varepsilon_{\Phi_{\rho}} g_{\Phi_{\rho}}(x, y, z, T)\right]$, where $D_{0 \Phi_{\rho}}$ are the average values of diffusion coefficients. In this situation the Eqs.(6) could be written as

$$
\begin{aligned}
& \frac{\partial \Phi_{I}(x, y, z, t)}{\partial t}=D_{0 \Phi I} \frac{\partial}{\partial x}\left\{\left[1+\varepsilon_{\Phi I} g_{\Phi I}(x, y, z, T)\right] \frac{\partial \Phi_{I}(x, y, z, t)}{\partial x}\right\}+k_{I, I}(x, y, z, T) I^{2}(x, y, z, t)+ \\
& +D_{0 \Phi I} \frac{\partial}{\partial y}\left\{\left[1+\varepsilon_{\Phi I} g_{\Phi I}(x, y, z, T)\right] \frac{\partial \Phi_{I}(x, y, z, t)}{\partial y}\right\}+D_{0 \Phi I} \frac{\partial}{\partial z}\left\{\left[1+\varepsilon_{\Phi I} g_{\Phi I}(x, y, z, T)\right] \frac{\partial \Phi_{I}(x, y, z, t)}{\partial z}\right\}- \\
& -k_{I}(x, y, z, T) I(x, y, z, t) \\
& \frac{\partial \Phi_{V}(x, y, z, t)}{\partial t}=D_{0 \Phi V} \frac{\partial}{\partial x}\left\{\left[1+\varepsilon_{\Phi V} g_{\Phi V}(x, y, z, T)\right] \frac{\partial \Phi_{V}(x, y, z, t)}{\partial x}\right\}+k_{I, I}(x, y, z, T) I^{2}(x, y, z, t)+ \\
& +D_{0 \Phi V} \frac{\partial}{\partial y}\left\{\left[1+\varepsilon_{\Phi V} g_{\Phi V}(x, y, z, T)\right] \frac{\partial \Phi_{V}(x, y, z, t)}{\partial y}\right\}+D_{0 \Phi V} \frac{\partial}{\partial z}\left\{\left[1+\varepsilon_{\Phi V} g_{\Phi V}(x, y, z, T)\right] \frac{\partial \Phi_{V}(x, y, z, t)}{\partial z}\right\}- \\
& -k_{I}(x, y, z, T) I(x, y, z, t) .
\end{aligned}
$$

Farther we determine solutions of above equations as the following power series

$$
\Phi_{\rho}(x, y, z, t)=\sum_{i=0}^{\infty} \varepsilon_{\Phi \rho}^{i} \Phi_{\rho i}(x, y, z, t)
$$

Now we used the series (11) into Eqs.(6) and appropriate boundary and initial conditions. The using gives the possibility to obtain equations for initial-order approximations of concentrations of complexes of defects $\Phi_{\rho 0}(x, y, z, t)$, corrections for them $\Phi_{\rho i}(x, y, z, t)$ (for them $i \geq 1$ ) and boundary and initial conditions for them. We remove equations and conditions to the Appendix. Solutions of the equations have been calculated by standard approaches $[24,25]$ and presented in the Appendix. 
On Modelling of Mass Transport for Formulation of Conditions for Increasing Integration Rate of Elements Framework Current Comparator. an Approach to Optimize of Technological Process

Now we calculate distribution of concentration of dopant in space and time by using the approach, which was used for analysis of radiation defects. To use the approach we consider following transformation of approximation of dopant diffusion coefficient: $D_{L}(x, y, z, T)=D_{0 L}\left[1+\varepsilon_{L} g_{L}(x, y, z, T)\right]$, where $D_{0 L}$ is the average value of dopant diffusion coefficient, $0 \leq \varepsilon_{L}<1,\left|g_{L}(x, y, z, T)\right| \leq 1$. Farther we consider solution of Eq.(1) as the following series:

$$
C(x, y, z, t)=\sum_{i=0}^{\infty} \varepsilon_{L}^{i} \sum_{j=1}^{\infty} \xi^{j} C_{i j}(x, y, z, t) .
$$

Using the relation into Eq.(1) and conditions (2) leads to obtaining equations for the functions $C_{i j}(x, y, z, t)(i \geq 1, j \geq 1)$, boundary and initial conditions for them. The equations are presented in the Appendix. Solutions of the equations have been calculated by standard approaches (see, for example, $[24,25])$. The solutions are presented in the Appendix.

We analyzed distributions of concentrations of dopant and radiation defects in space and time analytically by using the second-order approximations on all parameters, which have been used in appropriate series. Usually the second-order approximations are enough good approximations to make qualitative analysis and to obtain quantitative results. All analytical results have been checked by numerical simulation.

\section{DISCUSSION}

In this section we analyzed spatio-temporal distributions of concentrations of dopants. Figs. 2 shows typical spatial distributions of concentrations of dopants in neighborhood of interfaces of heterostructures. We calculate these distributions of concentrations of dopants under the following condition: value of dopant diffusion coefficient in doped area is larger, than value of dopant diffusion coefficient in nearest areas. In this situation one can find increasing of compactness of field-effect transistors with increasing of homogeneity of distribution of concentration of dopant at one time. Changing relation between values of dopant diffusion coefficients leads to opposite result (see Figs. 3).

It should be noted, that framework the considered approach one shall optimize annealing of dopant and/or radiation defects. To do the optimization we used recently introduced criterion [26-34]. The optimization based on approximation real distribution by step-wise function $\psi(x, y, z)$ (see Figs. 4). Farther the required values of optimal annealing time have been calculated by minimization the following mean-squared error

$$
U=\frac{1}{L_{x} L_{y} L_{z}} \int_{0}^{L_{x}} \int_{0}^{L_{y}} \int_{0}^{L_{z}}[C(x, y, z, \Theta)-\psi(x, y, z)] d z d y d x .
$$

We show optimal values of annealing time as functions of parameters on Figs. 5. It is known, that standard step of manufactured ion-doped structures is annealing of radiation defects. In the ideal case after finishing the annealing dopant achieves interface between layers of heterostructure. If the dopant has no enough time to achieve the interface, it is practicably to anneal the dopant additionally. The Fig. $5 b$ shows the described dependences of optimal values of additional annealing time for the same parameters as for Fig. $5 a$. Necessity to anneal radiation defects leads to smaller values of optimal annealing of implanted dopant in comparison with optimal annealing time of infused dopant. 
On Modelling of Mass Transport for Formulation of Conditions for Increasing Integration Rate of Elements Framework Current Comparator. an Approach to Optimize of Technological Process

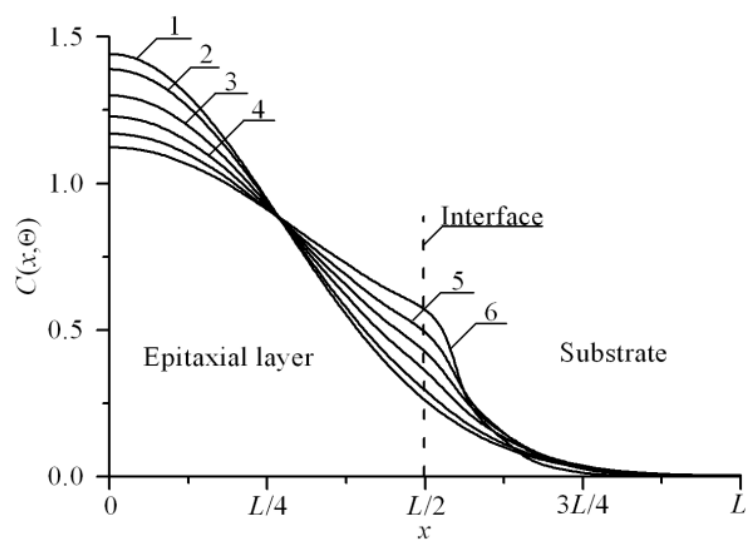

Fig2a. Dependences of concentration of dopant, infused in heterostructure from Figs. 1, on coordinate in direction, which is perpendicular to interface between epitaxial layer substrate. Difference between values of dopant diffusion coefficient in layers of heterostructure increases with increasing of number of curves. Value of dopant diffusion coefficient in the epitaxial layer is larger, than value of dopant diffusion coefficient in the substrate

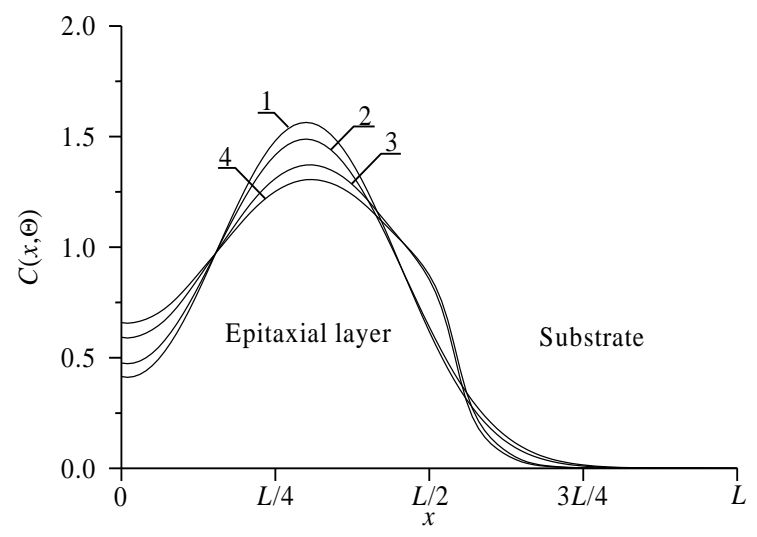

Fig2b. Dependences of concentration of dopant, implanted in heterostructure from Figs. 1, on coordinate in direction, which is perpendicular to interface between epitaxial layer substrate. Difference between values of dopant diffusion coefficient in layers of heterostructure increases with increasing of number of curves. Value of dopant diffusion coefficient in the epitaxial layer is larger, than value of dopant diffusion coefficient in the substrate. Curve 1 corresponds to homogenous sample and annealing time $\Theta=0.0048\left(L_{x}{ }^{2}+L_{y}{ }^{2}+L_{z}{ }^{2}\right) / D_{0}$. Curve 2 corresponds to homogenous sample and annealing time $\Theta=0.0057\left(L_{x}{ }^{2}+L_{y}{ }^{2}+L_{z}{ }^{2}\right) / D_{0}$. Curves 3 and 4 correspond to heterostructure from Figs. 1; annealing times $\Theta=0.0048\left(L_{x}{ }^{2}+L_{y}{ }^{2}+L_{z}{ }^{2}\right) / D_{0}$ and $\Theta=0.0057\left(L_{x}{ }^{2}\right.$ $\left.+L_{y}{ }^{2}+L_{z}{ }^{2}\right) / D_{0}$, respectively

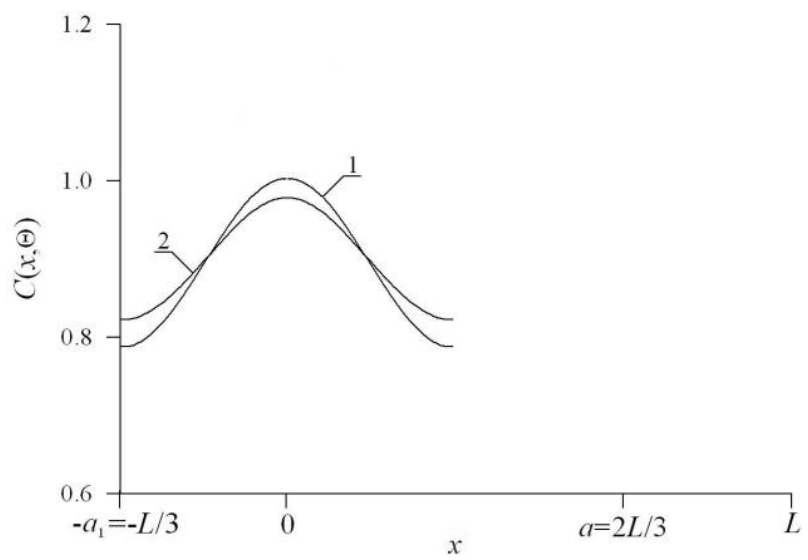

Fig3a. Distributions of concentration of dopant, infused in average section of epitaxial layer of heterostructure from Figs. 1 in direction parallel to interface between epitaxial layer and substrate of heterostructure. Difference between values of dopant diffusion coefficients increases with increasing of number of curves. Value of dopant diffusion coefficient in this section is smaller, than value of dopant diffusion coefficient in nearest sections 
On Modelling of Mass Transport for Formulation of Conditions for Increasing Integration Rate of Elements Framework Current Comparator. an Approach to Optimize of Technological Process

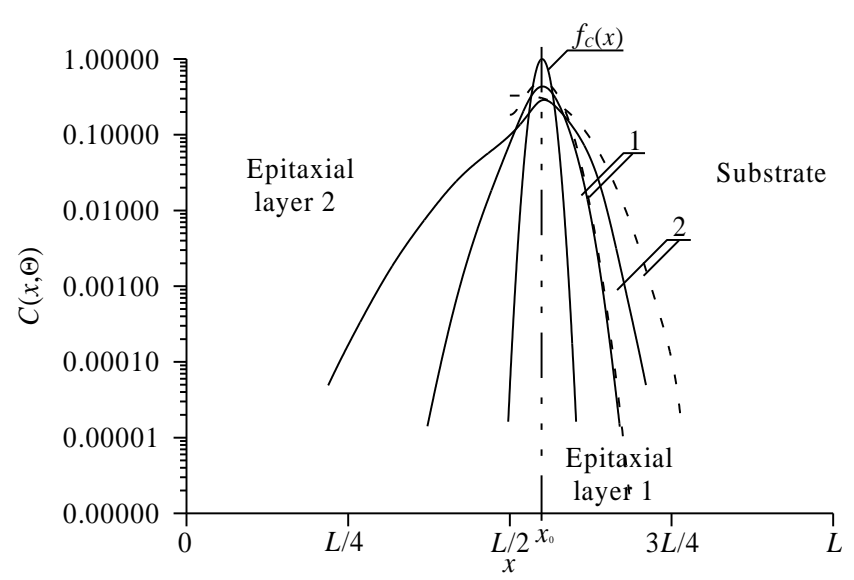

Fig3b. Calculated distributions of implanted dopant in epitaxial layers of heterostructure. Solid lines are spatial distributions of implanted dopant in system of two epitaxial layers. Dushed lines are spatial distributions of implanted dopant in one epitaxial layer. Annealing time increases with increasing of number of curves

\section{CONCLUSiOnS}

In this paper we introduce an approach to increase integration rate of element of a current comparator. The approach gives us possibility to decrease area of the elements with smaller increasing of the element's thickness.

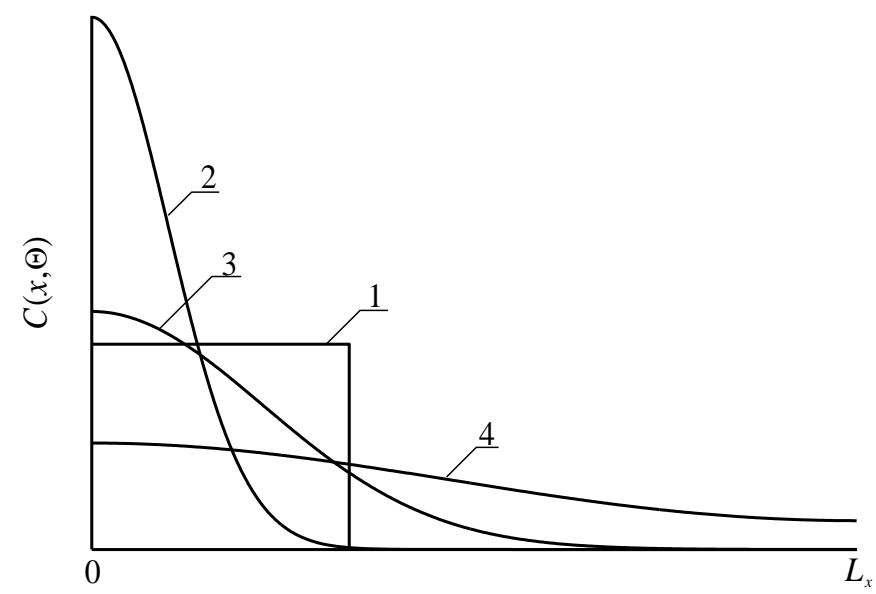

Fig4a. Distributions of concentration of infused dopant in depth of heterostructure from Fig. 1 for different values of annealing time (curves 2-4) and idealized step-wise approximation (curve 1). Increasing of number of curve corresponds to increasing of annealing time

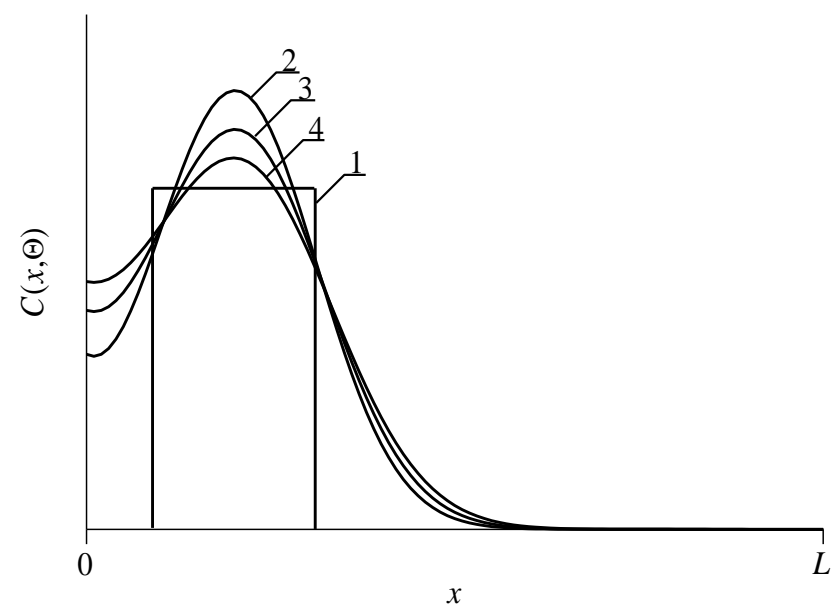

Fig4b. Distributions of concentration of implanted dopant in depth of heterostructure from Fig. 1 for different values of annealing time (curves 2-4) and idealized step-wise approximation (curve 1). Increasing of number of curve corresponds to increasing of annealing time 
On Modelling of Mass Transport for Formulation of Conditions for Increasing Integration Rate of Elements Framework Current Comparator. an Approach to Optimize of Technological Process

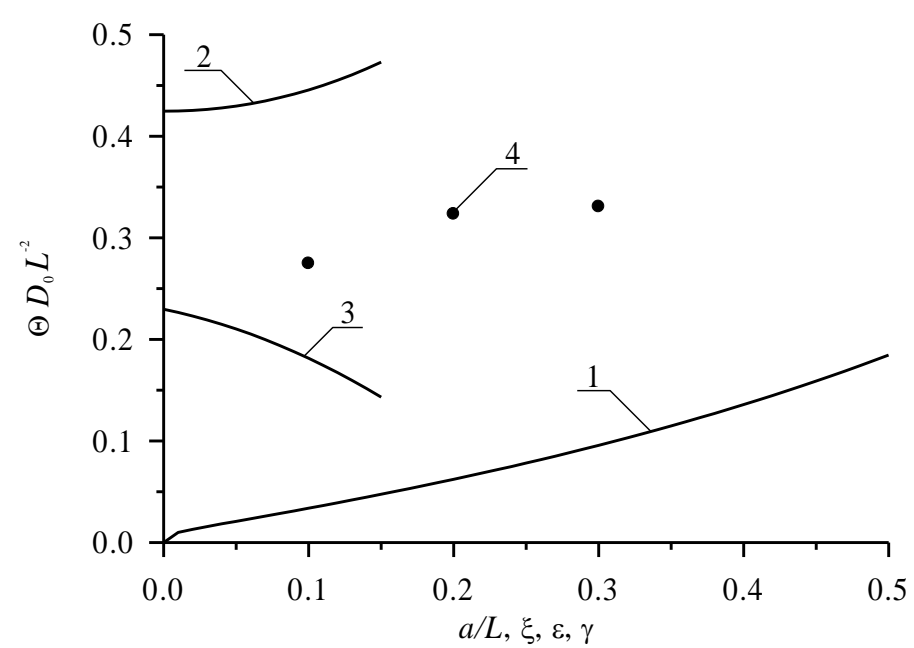

Fig5a. Dimensionless optimal annealing time of infused dopant as a function of several parameters. Curve 1 describes the dependence of the annealing time on the relation $a / L$ and $\xi=\gamma=0$ for equal to each other values of dopant diffusion coefficient in all parts of heterostructure. Curve 2 describes the dependence of the annealing time on value of parameter $\varepsilon$ for $a / L=1 / 2$ and $\xi=\gamma=0$. Curve 3 describes the dependence of the annealing time on value of parameter $\xi$ for $a / L=1 / 2$ and $\varepsilon=\gamma=0$. Curve 4 describes the dependence of the annealing time on value of parameter $\gamma$ for $a / L=1 / 2$ and $\varepsilon=\xi=0$

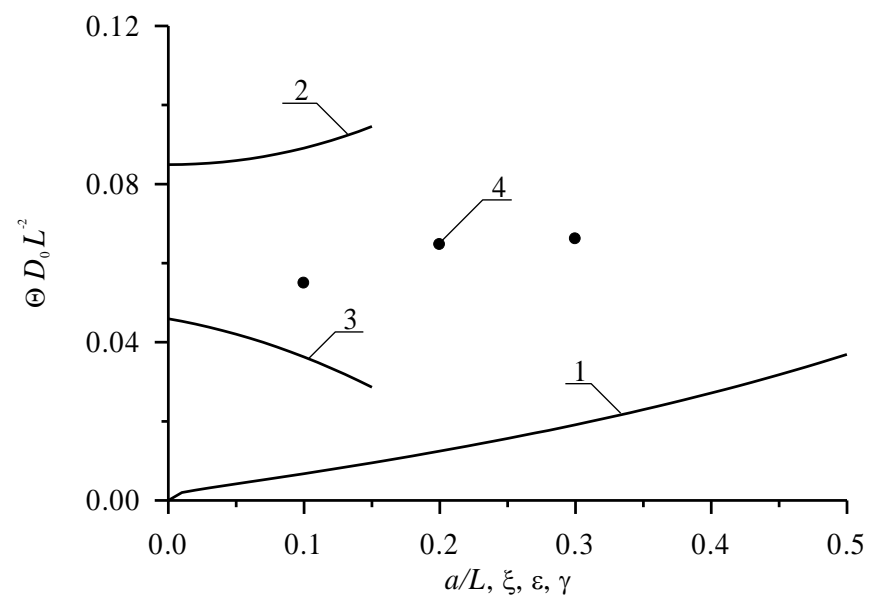

Fig5b. Dimensionless optimal annealing time of implanted dopant as a function of several parameters. Curve 1 describes the dependence of the annealing time on the relation $a / L$ and $\xi=\gamma=0$ for equal to each other values of dopant diffusion coefficient in all parts of heterostructure. Curve 2 describes the dependence of the annealing time on value of parameter $\varepsilon$ for $a / L=1 / 2$ and $\xi=\gamma=0$. Curve 3 describes the dependence of the annealing time on value of parameter $\xi$ for $a / L=1 / 2$ and $\varepsilon=\gamma=0$. Curve 4 describes the dependence of the annealing time on value of parameter $\gamma$ for $a / L=1 / 2$ and $\varepsilon=\xi=0$

\section{REFERENCES}

[1] V.I. Lachin, N.S. Savelov. Electronics (Phoenix, Rostov-na-Donu, 2001).

[2] A.G. Alexenko, I.I. Shagurin. Microcircuitry (Radio and communication, Moscow, 1990).

[3] N.A. Avaev, Yu.E. Naumov, V.T. Frolkin. Basis of microelectronics (Radio and communication, Moscow, 1991).

[4] A. Baradaranrezaeii, O. Shino, K. Hadidi, A. Khoei. Analog. Integr. Circ. Sig. Process. Vol. 85. P. 181192 (2015).

[5] D. Fathi, B. Forouzandeh, N. Masoumi. Nano. Vol. 4 (4). P. 233-238 (2009).

[6] S.A. Chachuli, P.N.A. Fasyar, N. Soin, N.M. Kar, N. Yusop. Mat. Sci. Sem. Proc. Vol. 24. P. 9-14 (2014).

[7] A.O. Ageev, A.E. Belyaev, N.S. Boltovets, V.N. Ivanov, R.V. Konakova, Ya.Ya. Kudrik, P.M. Litvin, V.V. Milenin, A.V. Sachenko. Semiconductors. Vol. 43 (7). P. 897-903 (2009). 
On Modelling of Mass Transport for Formulation of Conditions for Increasing Integration Rate of Elements Framework Current Comparator. an Approach to Optimize of Technological Process

[8] Z. Li, J. Waldron, T. Detchprohm, C. Wetzel, R.F. Karlicek, Jr.T.P. Chow. Appl. Phys. Lett. Vol. 102 (19). P. 192107-192109 (2013).

[9] Jung-Hui Tsai, Shao-Yen Chiu, Wen-Shiung Lour, Der-Feng Guo. Semiconductors. Vol. 43 (7). P. 971974 (2009).

[10] O.V. Alexandrov, A.O. Zakhar'in, N.A. Sobolev, E.I. Shek, M.M. Makoviychuk, E.O. Parshin. Semiconductors. Vol. 32 (9). P. 1029-1032 (1998).

[11] M.J. Kumar, T.V. Singh. Int. J. Nanoscience. Vol. 7 (2-3). P. 81-84 (2008).

[12] P. Sinsermsuksakul, K. Hartman, S.B. Kim, J. Heo, L. Sun, H.H. Park, R. Chakraborty, T. Buonassisi, R.G. Gordon. Appl. Phys. Lett. Vol. 102 (5). P. 053901-053905 (2013).

[13] J.G. Reynolds, C.L. Reynolds, Jr.A. Mohanta, J.F. Muth, J.E. Rowe, H.O. Everitt, D.E. Aspnes. Appl. Phys. Lett. Vol. 102 (15). P. 152114-152118 (2013).

[14] K.K. Ong, K.L. Pey, P.S. Lee, A.T.S. Wee, X.C. Wang, Y.F. Chong. Appl. Phys. Lett. Vol. 89 (17). P. 172111-172114 (2006).

[15] H.T. Wang, L.S. Tan, E. F. Chor. J. Appl. Phys. Vol. 98 (9). P. $094901-094905$ (2006).

[16] S.T. Shishiyanu, T.S. Shishiyanu, S.K. Railyan. Semiconductors. Vol. 36 (5). P. 611-617 (2002).

[17] Yu.V. Bykov, A.G. Yeremeev, N.A. Zharova, I.V. Plotnikov, K.I. Rybakov, M.N. Drozdov, Yu.N. Drozdov, V.D. Skupov. Radiophysics and Quantum Electronics. Vol. 43 (3). P. 836-843 (2003).

[18] E.L. Pankratov, E.A. Bulaeva. Reviews in Theoretical Science. Vol. 1 (1). P. 58-82 (2013).

[19] Yu.N. Erofeev. Pulse devices (Higher School, Moscow, 1989, in Russian).

[20] V.V. Kozlivsky. Modification of semiconductors by proton beams (Nauka, Sant-Peterburg, 2003, in Russian).

[21] Z.Yu. Gotra. Technology of microelectronic devices (Radio and communication, Moscow, 1991).

[22] V.L. Vinetskiy, G.A. Kholodar', Radiative physics of semiconductors. ("Naukova Dumka", Kiev, 1979, in Russian).

[23] P.M. Fahey, P.B. Griffin, J.D. Plummer. Rev. Mod. Phys. Vol. 61 (2). P. 289-388 (1989).

[24] A.N. Tikhonov, A.A. Samarskii. The mathematical physics equations (Moscow, Nauka 1972) (in Russian).

[25] H.S. Carslaw, J.C. Jaeger. Conduction of heat in solids (Oxford University Press, 1964).

[26] E.L. Pankratov. Russian Microelectronics. Vol. 36 (1). P. 33-39 (2007).

[27] E.L. Pankratov. Int. J. Nanoscience. Vol. 7 (4-5). P. 187-197 (2008).

[28] E.L. Pankratov. J. Comp. Theor. Nanoscience. Vol. 14 (10). P.4885-4899 (2017).

[29] E.L. Pankratov. Advanced science, engineering and medicine. Vol. 9 (9). P. 787 - 801 (2017).

[30] E.L. Pankratov, E.A. Bulaeva. Multidiscipline Modeling in Materials and Structures. Vol. 13 (4). P. 664677 (2017).

[31] E.L. Pankratov, E.A. Bulaeva. Int. J. Micro-Nano Scale Transp. Vol. 4 (1). P. 17-31 (2014).

[32] E.L. Pankratov, E.A. Bulaeva. Multidiscipline Modeling in Materials and Structures. Vol. 12 (4). P. 578604 (2016).

[33] E.L. Pankratov, E.A. Bulaeva. Materials science in semiconductor processing. Vol. 34. P. 260-268 (2015).

\section{APPENDIX}

Equations for the functions $\tilde{I}_{i j k}(\chi, \eta, \phi, \vartheta)$ and $\tilde{V}_{i j k}(\chi, \eta, \phi, \vartheta), i \geq 0, j \geq 0, k \geq 0$ and conditions for them

$$
\begin{aligned}
& \frac{\partial \tilde{I}_{000}(\chi, \eta, \phi, \vartheta)}{\partial \vartheta}=\sqrt{\frac{D_{0 I}}{D_{0 V}}}\left[\frac{\partial^{2} \tilde{I}_{000}(\chi, \eta, \phi, \vartheta)}{\partial \chi^{2}}+\frac{\partial^{2} \tilde{I}_{000}(\chi, \eta, \phi, \vartheta)}{\partial \eta^{2}}+\frac{\partial^{2} \tilde{I}_{000}(\chi, \eta, \phi, \vartheta)}{\partial \phi^{2}}\right] \\
& \frac{\partial \tilde{V}_{000}(\chi, \eta, \phi, \vartheta)}{\partial \vartheta}=\sqrt{\frac{D_{0 V}}{D_{0 I}}}\left[\frac{\partial^{2} \tilde{V}_{000}(\chi, \eta, \phi, \vartheta)}{\partial \chi^{2}}+\frac{\partial^{2} \tilde{V}_{000}(\chi, \eta, \phi, \vartheta)}{\partial \eta^{2}}+\frac{\partial^{2} \tilde{V}_{000}(\chi, \eta, \phi, \vartheta)}{\partial \phi^{2}}\right] ; \\
& \frac{\partial \tilde{I}_{i 00}(\chi, \vartheta)}{\partial \vartheta}=\sqrt{\frac{D_{0 I}}{D_{0 V}}}\left[\frac{\partial^{2} \tilde{I}_{i 00}(\chi, \eta, \phi, \vartheta)}{\partial \chi^{2}}+\frac{\partial^{2} \tilde{I}_{i 00}(\chi, \eta, \phi, \vartheta)}{\partial \eta^{2}}+\frac{\partial^{2} \tilde{I}_{i 00}(\chi, \eta, \phi, \vartheta)}{\partial \phi^{2}}\right]+\sqrt{\frac{D_{0 I}}{D_{0 V}}} \times
\end{aligned}
$$


On Modelling of Mass Transport for Formulation of Conditions for Increasing Integration Rate of Elements Framework Current Comparator. an Approach to Optimize of Technological Process

$$
\begin{aligned}
& \times\left\{\frac{\partial}{\partial \chi}\left[g_{I}(\chi, \eta, \phi, T) \frac{\partial \tilde{I}_{i-100}(\chi, \eta, \phi, \vartheta)}{\partial \chi}\right]+\frac{\partial}{\partial \eta}\left[g_{I}(\chi, \eta, \phi, T) \frac{\partial \tilde{I}_{i-100}(\chi, \eta, \phi, \vartheta)}{\partial \eta}\right]+\right. \\
& \left.+\frac{\partial}{\partial \phi}\left[g_{I}(\chi, \eta, \phi, T) \frac{\partial \tilde{I}_{i-100}(\chi, \eta, \phi, \vartheta)}{\partial \phi}\right]\right\}, i \geq 1, \\
& \frac{\partial \tilde{V}_{i 00}(\chi, \vartheta)}{\partial \vartheta}=\sqrt{\frac{D_{0 V}}{D_{0 I}}}\left[\frac{\partial^{2} \tilde{V}_{i 00}(\chi, \eta, \phi, \vartheta)}{\partial \chi^{2}}+\frac{\partial^{2} \tilde{V}_{i 00}(\chi, \eta, \phi, \vartheta)}{\partial \eta^{2}}+\frac{\partial^{2} \tilde{V}_{i 00}(\chi, \eta, \phi, \vartheta)}{\partial \phi^{2}}\right]+\frac{\partial}{\partial \chi}\left[g_{V}(\chi, \eta, \phi, T) \times\right.
\end{aligned}
$$$$
\left.\times \frac{\partial \tilde{V}_{i-100}(\chi, \eta, \phi, \vartheta)}{\partial \chi}\right] \sqrt{\frac{D_{0 V}}{D_{0 I}}}+\sqrt{\frac{D_{0 V}}{D_{0 I}}} \frac{\partial}{\partial \eta}\left[g_{V}(\chi, \eta, \phi, T) \frac{\partial \tilde{V}_{i-100}(\chi, \eta, \phi, \vartheta)}{\partial \eta}\right]+\frac{\partial}{\partial \phi}\left[g_{V}(\chi, \eta, \phi, T) \times\right.
$$$$
\left.\times \frac{\partial \tilde{V}_{i-100}(\chi, \eta, \phi, \vartheta)}{\partial \phi}\right] \sqrt{\frac{D_{0 V}}{D_{0 I}}}, i \geq 1,
$$$$
\frac{\partial \tilde{I}_{010}(\chi, \eta, \phi, \vartheta)}{\partial \vartheta}=\sqrt{\frac{D_{0 I}}{D_{0 V}}}\left[\frac{\partial^{2} \tilde{I}_{010}(\chi, \eta, \phi, \vartheta)}{\partial \chi^{2}}+\frac{\partial^{2} \tilde{I}_{010}(\chi, \eta, \phi, \vartheta)}{\partial \eta^{2}}+\frac{\partial^{2} \tilde{I}_{010}(\chi, \eta, \phi, \vartheta)}{\partial \phi^{2}}\right]-
$$

$-\left[1+\varepsilon_{I, V} g_{I, V}(\chi, \eta, \phi, T)\right] \tilde{I}_{000}(\chi, \eta, \phi, \vartheta) \tilde{V}_{000}(\chi, \eta, \phi, \vartheta)$

$\frac{\partial \tilde{V}_{010}(\chi, \eta, \phi, \vartheta)}{\partial \vartheta}=\sqrt{\frac{D_{0 V}}{D_{0 I}}}\left[\frac{\partial^{2} \tilde{V}_{010}(\chi, \eta, \phi, \vartheta)}{\partial \chi^{2}}+\frac{\partial^{2} \tilde{V}_{010}(\chi, \eta, \phi, \vartheta)}{\partial \eta^{2}}+\frac{\partial^{2} \tilde{V}_{010}(\chi, \eta, \phi, \vartheta)}{\partial \phi^{2}}\right]-$

$-\left[1+\varepsilon_{I, V} g_{I, V}(\chi, \eta, \phi, T)\right] \tilde{I}_{000}(\chi, \eta, \phi, \vartheta) \tilde{V}_{000}(\chi, \eta, \phi, \vartheta)$;

$\frac{\partial \tilde{I}_{020}(\chi, \eta, \phi, \vartheta)}{\partial \vartheta}=\sqrt{\frac{D_{0 I}}{D_{0 V}}}\left[\frac{\partial^{2} \tilde{I}_{020}(\chi, \eta, \phi, \vartheta)}{\partial \chi^{2}}+\frac{\partial^{2} \tilde{I}_{020}(\chi, \eta, \phi, \vartheta)}{\partial \eta^{2}}+\frac{\partial^{2} \tilde{I}_{020}(\chi, \eta, \phi, \vartheta)}{\partial \phi^{2}}\right]-$

$-\left[1+\varepsilon_{I, V} g_{I, V}(\chi, \eta, \phi, T)\right]\left[\tilde{I}_{010}(\chi, \eta, \phi, \vartheta) \tilde{V}_{000}(\chi, \eta, \phi, \vartheta)+\tilde{I}_{000}(\chi, \eta, \phi, \vartheta) \tilde{V}_{010}(\chi, \eta, \phi, \vartheta)\right]$

$\frac{\partial \tilde{V}_{020}(\chi, \eta, \phi, \vartheta)}{\partial \vartheta}=\sqrt{\frac{D_{0 I}}{D_{0 V}}}\left[\frac{\partial^{2} \tilde{V}_{020}(\chi, \eta, \phi, \vartheta)}{\partial \chi^{2}}+\frac{\partial^{2} \tilde{V}_{020}(\chi, \eta, \phi, \vartheta)}{\partial \eta^{2}}+\frac{\partial^{2} \tilde{V}_{020}(\chi, \eta, \phi, \vartheta)}{\partial \phi^{2}}\right]-$

$-\left[1+\varepsilon_{I, V} g_{I, V}(\chi, \eta, \phi, T)\right]\left[\tilde{I}_{010}(\chi, \eta, \phi, \vartheta) \tilde{V}_{000}(\chi, \eta, \phi, \vartheta)+\tilde{I}_{000}(\chi, \eta, \phi, \vartheta) \tilde{V}_{010}(\chi, \eta, \phi, \vartheta)\right]$;

$\frac{\partial \tilde{I}_{001}(\chi, \eta, \phi, \vartheta)}{\partial \vartheta}=\sqrt{\frac{D_{0 I}}{D_{0 V}}}\left[\frac{\partial^{2} \tilde{I}_{001}(\chi, \eta, \phi, \vartheta)}{\partial \chi^{2}}+\frac{\partial^{2} \tilde{I}_{001}(\chi, \eta, \phi, \vartheta)}{\partial \eta^{2}}+\frac{\partial^{2} \tilde{I}_{001}(\chi, \eta, \phi, \vartheta)}{\partial \phi^{2}}\right]-$

$-\left[1+\varepsilon_{I, I} g_{I, I}(\chi, \eta, \phi, T)\right] \tilde{I}_{000}^{2}(\chi, \eta, \phi, \vartheta)$

$\frac{\partial \tilde{V}_{001}(\chi, \eta, \phi, \vartheta)}{\partial \vartheta}=\sqrt{\frac{D_{0 V}}{D_{0 I}}}\left[\frac{\partial^{2} \tilde{V}_{001}(\chi, \eta, \phi, \vartheta)}{\partial \chi^{2}}+\frac{\partial^{2} \tilde{V}_{001}(\chi, \eta, \phi, \vartheta)}{\partial \eta^{2}}+\frac{\partial^{2} \tilde{V}_{001}(\chi, \eta, \phi, \vartheta)}{\partial \phi^{2}}\right]-$

$-\left[1+\varepsilon_{I, I} g_{I, I}(\chi, \eta, \phi, T)\right] \tilde{V}_{000}^{2}(\chi, \eta, \phi, \vartheta) ;$

$\frac{\partial \tilde{I}_{110}(\chi, \eta, \phi, \vartheta)}{\partial \vartheta}=\sqrt{\frac{D_{0 I}}{D_{0 V}}}\left[\frac{\partial^{2} \tilde{I}_{110}(\chi, \eta, \phi, \vartheta)}{\partial \chi^{2}}+\frac{\partial^{2} \tilde{I}_{110}(\chi, \eta, \phi, \vartheta)}{\partial \eta^{2}}+\frac{\partial^{2} \tilde{I}_{110}(\chi, \eta, \phi, \vartheta)}{\partial \phi^{2}}\right]+\sqrt{\frac{D_{0 I}}{D_{0 V}}} \times$

$\times\left\{\frac{\partial}{\partial \chi}\left[g_{I}(\chi, \eta, \phi, T) \frac{\partial \tilde{I}_{010}(\chi, \eta, \phi, \vartheta)}{\partial \chi}\right]+\frac{\partial}{\partial \eta}\left[g_{I}(\chi, \eta, \phi, T) \frac{\partial \tilde{I}_{010}(\chi, \eta, \phi, \vartheta)}{\partial \eta}\right]+\frac{\partial}{\partial \phi}\left[g_{I}(\chi, \eta, \phi, T) \times\right.\right.$ 
On Modelling of Mass Transport for Formulation of Conditions for Increasing Integration Rate of Elements Framework Current Comparator. an Approach to Optimize of Technological Process

$\left.\left.\times \frac{\partial \tilde{I}_{010}(\chi, \eta, \phi, \vartheta)}{\partial \phi}\right]\right\}-\left[\tilde{I}_{100}(\chi, \eta, \phi, \vartheta) \tilde{V}_{000}(\chi, \eta, \phi, \vartheta)+\tilde{I}_{000}(\chi, \eta, \phi, \vartheta) \tilde{V}_{100}(\chi, \eta, \phi, \vartheta)\right] \times$

$\times\left\lfloor 1+\varepsilon_{I, I} g_{I, I}(\chi, \eta, \phi, T)\right\rfloor$

$\frac{\partial \tilde{V}_{110}(\chi, \eta, \phi, \vartheta)}{\partial \vartheta}=\sqrt{\frac{D_{0 V}}{D_{0 I}}}\left[\frac{\partial^{2} \tilde{V}_{110}(\chi, \eta, \phi, \vartheta)}{\partial \chi^{2}}+\frac{\partial^{2} \tilde{V}_{110}(\chi, \eta, \phi, \vartheta)}{\partial \eta^{2}}+\frac{\partial^{2} \tilde{V}_{110}(\chi, \eta, \phi, \vartheta)}{\partial \phi^{2}}\right]+$

$+\sqrt{\frac{D_{0 V}}{D_{0 I}}}\left\{\frac{\partial}{\partial \chi}\left[g_{V}(\chi, \eta, \phi, T) \frac{\partial \tilde{V}_{010}(\chi, \eta, \phi, \vartheta)}{\partial \chi}\right]+\frac{\partial}{\partial \eta}\left[g_{V}(\chi, \eta, \phi, T) \frac{\partial \tilde{V}_{010}(\chi, \eta, \phi, \vartheta)}{\partial \eta}\right]+\right.$

$\left.+\frac{\partial}{\partial \phi}\left[g_{V}(\chi, \eta, \phi, T) \frac{\partial \tilde{V}_{010}(\chi, \eta, \phi, \vartheta)}{\partial \phi}\right]\right\}-\left[1+\varepsilon_{V, V} g_{V, V}(\chi, \eta, \phi, T)\right] \times$

$\times\left[\tilde{V}_{100}(\chi, \eta, \phi, \vartheta) \tilde{I}_{000}(\chi, \eta, \phi, \vartheta)+\tilde{V}_{000}(\chi, \eta, \phi, \vartheta) \tilde{I}_{100}(\chi, \eta, \phi, \vartheta)\right]$

$\frac{\partial \tilde{I}_{002}(\chi, \eta, \phi, \vartheta)}{\partial \vartheta}=\sqrt{\frac{D_{0 I}}{D_{0 V}}}\left[\frac{\partial^{2} \tilde{I}_{002}(\chi, \eta, \phi, \vartheta)}{\partial \chi^{2}}+\frac{\partial^{2} \tilde{I}_{002}(\chi, \eta, \phi, \vartheta)}{\partial \eta^{2}}+\frac{\partial^{2} \tilde{I}_{002}(\chi, \eta, \phi, \vartheta)}{\partial \phi^{2}}\right]-$

$-\left[1+\varepsilon_{I, I} g_{I, I}(\chi, \eta, \phi, T)\right] \tilde{I}_{001}(\chi, \eta, \phi, \vartheta) \tilde{I}_{000}(\chi, \eta, \phi, \vartheta)$

$\frac{\partial \tilde{V}_{002}(\chi, \eta, \phi, \vartheta)}{\partial \vartheta}=\sqrt{\frac{D_{0 V}}{D_{0 I}}}\left[\frac{\partial^{2} \tilde{V}_{002}(\chi, \eta, \phi, \vartheta)}{\partial \chi^{2}}+\frac{\partial^{2} \tilde{V}_{002}(\chi, \eta, \phi, \vartheta)}{\partial \eta^{2}}+\frac{\partial^{2} \tilde{V}_{002}(\chi, \eta, \phi, \vartheta)}{\partial \phi^{2}}\right]-$

$-\left[1+\varepsilon_{V, V} g_{V, V}(\chi, \eta, \phi, E)\right] \tilde{V}_{001}(\chi, \eta, \phi, \vartheta) \tilde{V}_{000}(\chi, \eta, \phi, \vartheta) ;$

$\frac{\partial \tilde{I}_{101}(\chi, \eta, \phi, \vartheta)}{\partial \vartheta}=\sqrt{\frac{D_{0 I}}{D_{0 V}}}\left[\frac{\partial^{2} \tilde{I}_{101}(\chi, \eta, \phi, \vartheta)}{\partial \chi^{2}}+\frac{\partial^{2} \tilde{I}_{101}(\chi, \eta, \phi, \vartheta)}{\partial \eta^{2}}+\frac{\partial^{2} \tilde{I}_{101}(\chi, \eta, \phi, \vartheta)}{\partial \phi^{2}}\right]+$

$+\sqrt{\frac{D_{0 I}}{D_{0 V}}}\left\{\frac{\partial}{\partial \chi}\left[g_{I}(\chi, \eta, \phi, T) \frac{\partial \tilde{I}_{001}(\chi, \eta, \phi, \vartheta)}{\partial \chi}\right]+\frac{\partial}{\partial \eta}\left[g_{I}(\chi, \eta, \phi, T) \frac{\partial \tilde{I}_{001}(\chi, \eta, \phi, \vartheta)}{\partial \eta}\right]+\right.$

$\left.+\frac{\partial}{\partial \phi}\left[g_{I}(\chi, \eta, \phi, T) \frac{\partial \tilde{I}_{001}(\chi, \eta, \phi, \vartheta)}{\partial \phi}\right]\right\}-\left[1+\varepsilon_{I} g_{I}(\chi, \eta, \phi, T)\right] \tilde{I}_{100}(\chi, \eta, \phi, \vartheta) \tilde{V}_{000}(\chi, \eta, \phi, \vartheta)$

$\frac{\partial \tilde{V}_{101}(\chi, \eta, \phi, \vartheta)}{\partial \vartheta}=\sqrt{\frac{D_{0 V}}{D_{0 I}}}\left[\frac{\partial^{2} \tilde{V}_{101}(\chi, \eta, \phi, \vartheta)}{\partial \chi^{2}}+\frac{\partial^{2} \tilde{V}_{101}(\chi, \eta, \phi, \vartheta)}{\partial \eta^{2}}+\frac{\partial^{2} \tilde{V}_{101}(\chi, \eta, \phi, \vartheta)}{\partial \phi^{2}}\right]+$

$+\sqrt{\frac{D_{0 V}}{D_{0 I}}}\left\{\frac{\partial}{\partial \chi}\left[g_{V}(\chi, \eta, \phi, T) \frac{\partial \tilde{V}_{001}(\chi, \eta, \phi, \vartheta)}{\partial \chi}\right]+\frac{\partial}{\partial \eta}\left[g_{V}(\chi, \eta, \phi, T) \frac{\partial \tilde{V}_{001}(\chi, \eta, \phi, \vartheta)}{\partial \eta}\right]+\right.$

$\left.+\frac{\partial}{\partial \phi}\left[g_{V}(\chi, \eta, \phi, T) \frac{\partial \tilde{V}_{001}(\chi, \eta, \phi, \vartheta)}{\partial \phi}\right]\right\}-\left[1+\varepsilon_{V} g_{V}(\chi, \eta, \phi, T)\right] \tilde{I}_{000}(\chi, \eta, \phi, \vartheta) \tilde{V}_{100}(\chi, \eta, \phi, \vartheta)$;

$\frac{\partial \tilde{I}_{011}(\chi, \eta, \phi, \vartheta)}{\partial \vartheta}=\sqrt{\frac{D_{0 I}}{D_{0 V}}}\left[\frac{\partial^{2} \tilde{I}_{011}(\chi, \eta, \phi, \vartheta)}{\partial \chi^{2}}+\frac{\partial^{2} \tilde{I}_{011}(\chi, \eta, \phi, \vartheta)}{\partial \eta^{2}}+\frac{\partial^{2} \tilde{I}_{011}(\chi, \eta, \phi, \vartheta)}{\partial \phi^{2}}\right]-\tilde{I}_{010}(\chi, \eta, \phi, \vartheta) \times$

$\times\left[1+\varepsilon_{I, I} g_{I, I}(\chi, \eta, \phi, T)\right] \tilde{I}_{000}(\chi, \eta, \phi, \vartheta)-\left[1+\varepsilon_{I, V} g_{I, V}(\chi, \eta, \phi, T)\right] \tilde{I}_{001}(\chi, \eta, \phi, \vartheta) \tilde{V}_{000}(\chi, \eta, \phi, \vartheta)$ 
On Modelling of Mass Transport for Formulation of Conditions for Increasing Integration Rate of Elements Framework Current Comparator. an Approach to Optimize of Technological Process

$\frac{\partial \tilde{V}_{011}(\chi, \eta, \phi, \vartheta)}{\partial \vartheta}=\sqrt{\frac{D_{0 V}}{D_{0 I}}}\left[\frac{\partial^{2} \tilde{V}_{011}(\chi, \eta, \phi, \vartheta)}{\partial \chi^{2}}+\frac{\partial^{2} \tilde{V}_{011}(\chi, \eta, \phi, \vartheta)}{\partial \eta^{2}}+\frac{\partial^{2} \tilde{V}_{011}(\chi, \eta, \phi, \vartheta)}{\partial \phi^{2}}\right]-\tilde{V}_{010}(\chi, \eta, \phi, \vartheta) \times$

$\times\left[1+\varepsilon_{V, V} g_{V, V}(\chi, \eta, \phi, T)\right] \tilde{V}_{000}(\chi, \eta, \phi, \vartheta)-\left[1+\varepsilon_{I, V} g_{I, V}(\chi, \eta, \phi, t)\right] \tilde{I}_{000}(\chi, \eta, \phi, \vartheta) \tilde{V}_{001}(\chi, \eta, \phi, \vartheta) ;$

$\left.\frac{\partial \tilde{\rho}_{i j k}(\chi, \eta, \phi, \vartheta)}{\partial \chi}\right|_{x=0}=0,\left.\frac{\partial \tilde{\rho}_{i j k}(\chi, \eta, \phi, \vartheta)}{\partial \chi}\right|_{x=1}=0,\left.\frac{\partial \tilde{\rho}_{i j k}(\chi, \eta, \phi, \vartheta)}{\partial \eta}\right|_{\eta=0}=0,\left.\frac{\partial \tilde{\rho}_{i j k}(\chi, \eta, \phi, \vartheta)}{\partial \eta}\right|_{\eta=1}=0$,

$\left.\frac{\partial \tilde{\rho}_{i j k}(\chi, \eta, \phi, \vartheta)}{\partial \phi}\right|_{\phi=0}=0,\left.\frac{\partial \tilde{\rho}_{i j k}(\chi, \eta, \phi, \vartheta)}{\partial \phi}\right|_{\phi=1}=0(i \geq 0, j \geq 0, k \geq 0)$;

$\tilde{\rho}_{000}(\chi, \eta, \phi, 0)=f_{\rho}(\chi, \eta, \phi) / \rho^{*}, \tilde{\rho}_{i j k}(\chi, \eta, \phi, 0)=0(i \geq 1, j \geq 1, k \geq 1)$.

Solutions of the above equations could be written as

$$
\tilde{\rho}_{000}(\chi, \eta, \phi, \vartheta)=\frac{1}{L}+\frac{2}{L} \sum_{n=1}^{\infty} F_{n \rho} c(\chi) c(\eta) c(\phi) e_{n \rho}(\vartheta)
$$

where $F_{n \rho}=\frac{1}{\rho^{*}} \int_{0}^{1} \cos (\pi n u) \int_{0}^{1} \cos (\pi n v) \int_{0}^{1} \cos (\pi n w) f_{n \rho}(u, v, w) d w d v d u, c_{n}(\chi)=\cos (\pi \quad n \quad \chi)$, $e_{n I}(\vartheta)=\exp \left(-\pi^{2} n^{2} \vartheta \sqrt{D_{0 V} / D_{0 I}}\right), e_{n V}(\vartheta)=\exp \left(-\pi^{2} n^{2} \vartheta \sqrt{D_{0 I} / D_{0 V}}\right)$

$\tilde{I}_{i 00}(\chi, \eta, \phi, \vartheta)=-2 \pi \sqrt{\frac{D_{0 I}}{D_{0 V}}} \sum_{n=1}^{\infty} n c_{n}(\chi) c(\eta) c(\phi) e_{n I}(\vartheta) \int_{0}^{\vartheta} e_{n I}(-\tau) \int_{0}^{1} s_{n}(u) \int_{0}^{1} c_{n}(v) \int_{0}^{1} \frac{\partial \tilde{I}_{i-100}(u, v, w, \tau)}{\partial u} \times$

$\times c_{n}(w) g_{I}(u, v, w, T) d w d v d u d \tau-2 \pi \sqrt{\frac{D_{0 I}}{D_{0 V}}} \sum_{n=1}^{\infty} n c_{n}(\chi) c(\eta) c(\phi) e_{n I}(\vartheta) \int_{0}^{\vartheta} e_{n I}(-\tau) \int_{0}^{1} c_{n}(u) \int_{0}^{1} s_{n}(v) \times$

$\times \int_{0}^{1} c_{n}(w) g_{I}(u, v, w, T) \frac{\partial \tilde{I}_{i-100}(u, v, w, \tau)}{\partial v} d w d v d u d \tau-2 \pi \sqrt{\frac{D_{0 I}}{D_{0 V}}} \sum_{n=1}^{\infty} n c_{n}(\chi) c(\eta) c(\phi) e_{n I}(\vartheta) \int_{0}^{\vartheta} e_{n I}(-\tau) \times$

$\times \int_{0}^{1} c_{n}(u) \int_{0}^{1} c_{n}(v) \int_{0}^{1} s_{n}(w) g_{I}(u, v, w, T) \frac{\partial \tilde{I}_{i-100}(u, v, w, \tau)}{\partial w} d w d v d u d \tau, i \geq 1$,

$\tilde{V}_{i 00}(\chi, \eta, \phi, \vartheta)=-2 \pi \sqrt{\frac{D_{0 V}}{D_{0 I}}} \sum_{n=1}^{\infty} n c_{n}(\chi) c(\eta) c(\phi) e_{n V}(\vartheta) \int_{0}^{\vartheta} e_{n I}(-\tau) \int_{0}^{1} s_{n}(u) \int_{0}^{1} c_{n}(v) \int_{0}^{1} g_{V}(u, v, w, T) \times$

$\times c_{n}(w) \frac{\partial \tilde{V}_{i-100}(u, \tau)}{\partial u} d w d v d u d \tau-\sqrt{\frac{D_{0 V}}{D_{0 I}}} \sum_{n=1}^{\infty} n c_{n}(\chi) c(\eta) c(\phi) e_{n V}(\vartheta) \int_{0}^{\vartheta} e_{n I}(-\tau) \int_{0}^{1} c_{n}(u) \int_{0}^{1} s_{n}(v) \times$

$\times 2 \pi \int_{0}^{1} c_{n}(w) g_{V}(u, v, w, T) \frac{\partial \tilde{V}_{i-100}(u, \tau)}{\partial v} d w d v d u d \tau-2 \pi \sqrt{\frac{D_{0 V}}{D_{0 I}}} \sum_{n=1}^{\infty} n c_{n}(\chi) c(\eta) c(\phi) e_{n V}(\vartheta) \times$

$\times \int_{0}^{\vartheta} e_{n I}(-\tau) \int_{0}^{1} c_{n}(u) \int_{0}^{1} c_{n}(v) \int_{0}^{1} s_{n}(w) g_{V}(u, v, w, T) \frac{\partial \tilde{V}_{i-100}(u, \tau)}{\partial w} d w d v d u d \tau, i \geq 1$,

where $s_{n}(\chi)=\sin (\pi n \chi)$;

$\tilde{\rho}_{010}(\chi, \eta, \phi, \vartheta)=-2 \sum_{n=1}^{\infty} c_{n}(\chi) c_{n}(\eta) c_{n}(\phi) e_{n \rho}(\vartheta) \int_{0}^{\vartheta} e_{n \rho}(-\tau) \int_{0}^{1} c_{n}(u) \int_{0}^{1} c_{n}(v) \int_{0}^{1} c_{n}(w) \times$

$\times\left[1+\varepsilon_{I, V} g_{I, V}(u, v, w, T)\right] \tilde{I}_{000}(u, v, w, \tau) \tilde{V}_{000}(u, v, w, \tau) d w d v d u d \tau$ 
On Modelling of Mass Transport for Formulation of Conditions for Increasing Integration Rate of Elements Framework Current Comparator. an Approach to Optimize of Technological Process

$\tilde{\rho}_{020}(\chi, \eta, \phi, \vartheta)=-2 \sqrt{\frac{D_{0 I}}{D_{0 V}}} \sum_{n=1}^{\infty} c_{n}(\chi) c_{n}(\eta) c_{n}(\phi) e_{n \rho}(\vartheta) \int_{0}^{\vartheta} e_{n \rho}(-\tau) \int_{0}^{1} c_{n}(u) \int_{0}^{1} c_{n}(v) \int_{0}^{1} c_{n}(w)\left[1+\varepsilon_{I, V} \times\right.$

$\left.\times g_{I, V}(u, v, w, T)\right]\left[\tilde{I}_{010}(u, v, w, \tau) \tilde{V}_{000}(u, v, w, \tau)+\tilde{I}_{000}(u, v, w, \tau) \tilde{V}_{010}(u, v, w, \tau)\right] d w d v d u d \tau$;

$\tilde{\rho}_{001}(\chi, \eta, \phi, \vartheta)=-2 \sum_{n=1}^{\infty} c_{n}(\chi) c_{n}(\eta) c_{n}(\phi) e_{n \rho}(\vartheta) \int_{0}^{\vartheta} e_{n \rho}(-\tau) \int_{0}^{1} c_{n}(u) \int_{0}^{1} c_{n}(v) \int_{0}^{1} c_{n}(w) \times$

$\times\left[1+\varepsilon_{\rho, \rho} g_{\rho, \rho}(u, v, w, T)\right] \tilde{\rho}_{000}^{2}(u, v, w, \tau) d w d v d u d \tau$;

$\tilde{\rho}_{002}(\chi, \eta, \phi, \vartheta)=-2 \sum_{n=1}^{\infty} c_{n}(\chi) c_{n}(\eta) c_{n}(\phi) e_{n \rho}(\vartheta) \int_{0}^{\vartheta} e_{n \rho}(-\tau) \int_{0}^{1} c_{n}(u) \int_{0}^{1} c_{n}(v) \int_{0}^{1} c_{n}(w) \times$

$\times\left[1+\varepsilon_{\rho, \rho} g_{\rho, \rho}(u, v, w, T)\right] \tilde{\rho}_{001}(u, v, w, \tau) \tilde{\rho}_{000}(u, v, w, \tau) d w d v d u d \tau ;$

$\tilde{I}_{110}(\chi, \eta, \phi, \vartheta)=-2 \pi \sqrt{\frac{D_{0 I}}{D_{0 V}}} \sum_{n=1}^{\infty} n c_{n}(\chi) c_{n}(\eta) c_{n}(\phi) e_{n I}(\vartheta) \int_{0}^{\vartheta} e_{n I}(-\tau) \int_{0}^{1} s_{n}(u) \int_{0}^{1} c_{n}(v) \int_{0}^{1} c_{n}(u) \times$

$\times g_{I}(u, v, w, T) \frac{\partial \tilde{I}_{i-100}(u, v, w, \tau)}{\partial u} d w d v d u d \tau-2 \pi \sqrt{\frac{D_{0 I}}{D_{0 V}}} \sum_{n=1}^{\infty} n c_{n}(\chi) c_{n}(\eta) c_{n}(\phi) e_{n I}(\vartheta) \times$

$\times \int_{0}^{\vartheta} e_{n I}(-\tau) \int_{0}^{1} c_{n}(u) \int_{0}^{1} s_{n}(v) \int_{0}^{1} c_{n}(u) g_{I}(u, v, w, T) \frac{\partial \tilde{I}_{i-100}(u, v, w, \tau)}{\partial v} d w d v d u d \tau-2 \pi \sqrt{\frac{D_{0 I}}{D_{0 V}}} \times$

$\times \sum_{n=1}^{\infty} n e_{n I}(\vartheta) \int_{0}^{\vartheta} e_{n I}(-\tau) \int_{0}^{1} c_{n}(u) \int_{0}^{1} c_{n}(v) \int_{0}^{1} s_{n}(u) g_{I}(u, v, w, T) \frac{\partial \tilde{I}_{i-100}(u, v, w, \tau)}{\partial w} d w d v d u d \tau \times$

$\times c_{n}(\chi) c_{n}(\eta) c_{n}(\phi)-2 \sum_{n=1}^{\infty} c_{n}(\chi) e_{n I}(\vartheta) c_{n}(\eta) c_{n}(\phi) \int_{0}^{\vartheta} e_{n I}(-\tau) \int_{0}^{1} c_{n}(u) \int_{0}^{1} c_{n}(v) \int_{0}^{1} c_{n}(v)\left[1+\varepsilon_{I, V} \times\right.$

$\left.\times g_{I, V}(u, v, w, T)\right]\left[\tilde{I}_{100}(u, v, w, \tau) \tilde{V}_{000}(u, v, w, \tau)+\tilde{I}_{000}(u, v, w, \tau) \tilde{V}_{100}(u, v, w, \tau)\right] d w d v d u d \tau$

$\tilde{V}_{110}(\chi, \eta, \phi, \vartheta)=-2 \pi \sqrt{\frac{D_{0 V}}{D_{0 I}}} \sum_{n=1}^{\infty} n c_{n}(\chi) c_{n}(\eta) c_{n}(\phi) e_{n V}(\vartheta) \int_{0}^{\vartheta} e_{n V}(-\tau) \int_{0}^{1} s_{n}(u) \int_{0}^{1} c_{n}(v) \int_{0}^{1} c_{n}(u) \times$

$\times g_{V}(u, v, w, T) \frac{\partial \tilde{V}_{i-100}(u, v, w, \tau)}{\partial u} d w d v d u d \tau-2 \pi \sqrt{\frac{D_{0 V}}{D_{0 I}}} \sum_{n=1}^{\infty} n c_{n}(\chi) c_{n}(\eta) c_{n}(\phi) e_{n V}(\vartheta) \times$

$\times \int_{0}^{\vartheta} e_{n V}(-\tau) \int_{0}^{1} c_{n}(u) \int_{0}^{1} s_{n}(v) \int_{0}^{1} c_{n}(u) g_{V}(u, v, w, T) \frac{\partial \tilde{V}_{i-100}(u, v, w, \tau)}{\partial v} d w d v d u d \tau-2 \pi \sqrt{\frac{D_{0 V}}{D_{0 I}}} \times$

$\times \sum_{n=1}^{\infty} n e_{n V}(\vartheta) \int_{0}^{\vartheta} e_{n V}(-\tau) \int_{0}^{1} c_{n}(u) \int_{0}^{1} c_{n}(v) \int_{0}^{1} s_{n}(u) g_{V}(u, v, w, T) \frac{\partial \tilde{V}_{i-100}(u, v, w, \tau)}{\partial w} d w d v d u d \tau \times$

$\times c_{n}(\chi) c_{n}(\eta) c_{n}(\phi)-2 \sum_{n=1}^{\infty} c_{n}(\chi) e_{n I}(\vartheta) c_{n}(\eta) c_{n}(\phi) \int_{0}^{\vartheta} e_{n V}(-\tau) \int_{0}^{1} c_{n}(u) \int_{0}^{1} c_{n}(v) \int_{0}^{1}\left[1+\varepsilon_{I, V} g_{I, V}(u, v, w, T)\right] \times$

$\times c_{n}(w)\left[\tilde{I}_{100}(u, v, w, \tau) \tilde{V}_{000}(u, v, w, \tau)+\tilde{I}_{000}(u, v, w, \tau) \tilde{V}_{100}(u, v, w, \tau)\right] d w d v d u d \tau ;$

$\tilde{I}_{101}(\chi, \eta, \phi, \vartheta)=-2 \pi \sqrt{\frac{D_{0 I}}{D_{0 V}}} \sum_{n=1}^{\infty} n c_{n}(\chi) c_{n}(\eta) c_{n}(\phi) e_{n I}(\vartheta) \int_{0}^{\vartheta} e_{n I}(-\tau) \int_{0}^{1} s_{n}(u) \int_{0}^{1} c_{n}(v) \int_{0}^{1} g_{I}(u, v, w, T) \times$ 
On Modelling of Mass Transport for Formulation of Conditions for Increasing Integration Rate of Elements Framework Current Comparator. an Approach to Optimize of Technological Process

$\times c_{n}(w) \frac{\partial \tilde{I}_{001}(u, v, w, \tau)}{\partial u} d w d v d u d \tau-2 \pi \sqrt{\frac{D_{0 I}}{D_{0 V}}} \sum_{n=1}^{\infty} n c_{n}(\chi) c_{n}(\eta) c_{n}(\phi) e_{n I}(\vartheta) \times$

$\times \int_{0}^{1} s_{n}(v) \int_{0}^{1} c_{n}(w) g_{I}(u, v, w, T) \frac{\partial \tilde{I}_{001}(u, v, w, \tau)}{\partial v} d w d v d u d \tau-2 \pi \sqrt{\frac{D_{0 I}}{D_{0 V}}} \sum_{n=1}^{\infty} n e_{n I}(\vartheta) c_{n}(\chi) c_{n}(\eta) c_{n}(\phi) \times$

$\times \int_{0}^{\vartheta} e_{n I}(-\tau) \int_{0}^{1} c_{n}(u) \int_{0}^{1} c_{n}(v) \int_{0}^{1} s_{n}(w) g_{I}(u, v, w, T) \frac{\partial \tilde{I}_{001}(u, v, w, \tau)}{\partial w} d w d v d u d \tau-2 \sum_{n=1}^{\infty} c_{n}(\chi) c_{n}(\eta) c_{n}(\phi) \times$

$\times e_{n I}(\vartheta) \int_{0}^{\vartheta} e_{n I}(-\tau) \int_{0}^{1} c_{n}(u) \int_{0}^{1} c_{n}(v) \int_{0}^{1} c_{n}(w)\left[1+\varepsilon_{I, V} g_{I, V}(u, v, w, T)\right] \tilde{I}_{100}(u, v, w, \tau) \tilde{V}_{000}(u, v, w, \tau) d w d v d u d \tau$

$\tilde{V}_{101}(\chi, \eta, \phi, \vartheta)=-2 \pi \sqrt{\frac{D_{0 V}}{D_{0 I}}} \sum_{n=1}^{\infty} n c_{n}(\chi) c_{n}(\eta) c_{n}(\phi) e_{n V}(\vartheta) \int_{0}^{\vartheta} e_{n V}(-\tau) \int_{0}^{1} s_{n}(u) \int_{0}^{1} c_{n}(v) \int_{0}^{1} g_{V}(u, v, w, T) \times$

$\times c_{n}(w) \frac{\partial \tilde{V}_{001}(u, v, w, \tau)}{\partial u} d w d v d u d \tau-2 \pi \sqrt{\frac{D_{0 V}}{D_{0 I}}} \sum_{n=1}^{\infty} n c_{n}(\chi) c_{n}(\eta) c_{n}(\phi) e_{n I}(\vartheta) \int_{0}^{\vartheta} e_{n V}(-\tau) \int_{0}^{1} c_{n}(u) \times$

$\times \int_{0}^{1} s_{n}(v) \int_{0}^{1} c_{n}(w) g_{I}(u, v, w, T) \frac{\partial \tilde{I}_{001}(u, v, w, \tau)}{\partial v} d w d v d u d \tau-2 \pi \sqrt{\frac{D_{0 I}}{D_{0 V}}} \sum_{n=1}^{\infty} n e_{n I}(\vartheta) c_{n}(\chi) c_{n}(\eta) c_{n}(\phi) \times$

$\times \int_{0}^{\vartheta} e_{n V}(-\tau) \int_{0}^{1} c_{n}(u) \int_{0}^{1} c_{n}(v) \int_{0}^{1} s_{n}(w) g_{V}(u, v, w, T) \frac{\partial \tilde{V}_{001}(u, v, w, \tau)}{\partial w} d w d v d u d \tau-2 \sum_{n=1}^{\infty} c_{n}(\chi) c_{n}(\eta) c_{n}(\phi) \times$

$\times e_{n V}(\vartheta) \int_{0}^{\vartheta} e_{n V}(-\tau) \int_{0}^{1} c_{n}(u) \int_{0}^{1} c_{n}(v) \int_{0}^{1} c_{n}(w)\left[1+\varepsilon_{I, V} g_{I, V}(u, v, w, T)\right] \tilde{I}_{100}(u, v, w, \tau) \tilde{V}_{000}(u, v, w, \tau) d w d v d u d \tau ;$

$\tilde{I}_{011}(\chi, \eta, \phi, \vartheta)=-2 \sum_{n=1}^{\infty} c_{n}(\chi) c_{n}(\eta) c_{n}(\phi) e_{n I}(\vartheta) \int_{0}^{\vartheta} e_{n I}(-\tau) \int_{0}^{1} c_{n}(u) \int_{0}^{1} c_{n}(v) \int_{0}^{1} c_{n}(w)\left\{\tilde{I}_{000}(u, v, w, \tau) \times\right.$

$\left.\times\left[1+\varepsilon_{I, I} g_{I, I}(u, v, w, T)\right] \tilde{I}_{010}(u, v, w, \tau)+\left[1+\varepsilon_{I, V} g_{I, V}(u, v, w, T)\right] \tilde{I}_{001}(u, v, w, \tau) \tilde{V}_{000}(u, v, w, \tau)\right\} d w d v d u d \tau$

$\tilde{V}_{011}(\chi, \eta, \phi, \vartheta)=-2 \sum_{n=1}^{\infty} c_{n}(\chi) c_{n}(\eta) c_{n}(\phi) e_{n V}(\vartheta) \int_{0}^{\vartheta} e_{n V}(-\tau) \int_{0}^{1} c_{n}(u) \int_{0}^{1} c_{n}(v) \int_{0}^{1} c_{n}(w)\left\{\tilde{I}_{000}(u, v, w, \tau) \times\right.$

$\left.\times\left[1+\varepsilon_{I, I} g_{I, I}(u, v, w, T)\right] \tilde{I}_{010}(u, v, w, \tau)+\left[1+\varepsilon_{I, V} g_{I, V}(u, v, w, T)\right] \tilde{I}_{001}(u, v, w, \tau) \tilde{V}_{000}(u, v, w, \tau)\right\} d w d v d u d \tau$.

Equations for functions $\Phi_{\rho i}(x, y, z, t), i \geq 0$ to describe concentrations of simplest complexes of radiation defects.

$$
\begin{aligned}
& \frac{\partial \Phi_{I 0}(x, y, z, t)}{\partial t}=D_{0 \Phi I}\left[\frac{\partial^{2} \Phi_{I 0}(x, y, z, t)}{\partial x^{2}}+\frac{\partial^{2} \Phi_{I 0}(x, y, z, t)}{\partial y^{2}}+\frac{\partial^{2} \Phi_{I 0}(x, y, z, t)}{\partial z^{2}}\right]+ \\
& +k_{I, I}(x, y, z, T) I^{2}(x, y, z, t)-k_{I}(x, y, z, T) I(x, y, z, t) \\
& \frac{\partial \Phi_{V 0}(x, y, z, t)}{\partial t}=D_{0 \Phi V}\left[\frac{\partial^{2} \Phi_{V 0}(x, y, z, t)}{\partial x^{2}}+\frac{\partial^{2} \Phi_{V 0}(x, y, z, t)}{\partial y^{2}}+\frac{\partial^{2} \Phi_{V 0}(x, y, z, t)}{\partial z^{2}}\right]+ \\
& +k_{V, V}(x, y, z, T) V^{2}(x, y, z, t)-k_{V}(x, y, z, T) V(x, y, z, t) ; \\
& \frac{\partial \Phi_{I i}(x, y, z, t)}{\partial t}=D_{0 \Phi I}\left[\frac{\partial^{2} \Phi_{I i}(x, y, z, t)}{\partial x^{2}}+\frac{\partial^{2} \Phi_{I i}(x, y, z, t)}{\partial y^{2}}+\frac{\partial^{2} \Phi_{I i}(x, y, z, t)}{\partial z^{2}}\right]+
\end{aligned}
$$


On Modelling of Mass Transport for Formulation of Conditions for Increasing Integration Rate of Elements Framework Current Comparator. an Approach to Optimize of Technological Process

$$
\begin{aligned}
& +D_{0 \Phi I}\left\{\frac{\partial}{\partial x}\left[g_{\Phi I}(x, y, z, T) \frac{\partial \Phi_{I i-1}(x, y, z, t)}{\partial x}\right]+\frac{\partial}{\partial y}\left[g_{\Phi I}(x, y, z, T) \frac{\partial \Phi_{I i-1}(x, y, z, t)}{\partial y}\right]+\right. \\
& \left.+\frac{\partial}{\partial z}\left[g_{\Phi I}(x, y, z, T) \frac{\partial \Phi_{I i-1}(x, y, z, t)}{\partial z}\right]\right\}, i \geq 1, \\
& \frac{\partial \Phi_{V i}(x, y, z, t)}{\partial t}=D_{0 \Phi V}\left[\frac{\partial^{2} \Phi_{V i}(x, y, z, t)}{\partial x^{2}}+\frac{\partial^{2} \Phi_{V i}(x, y, z, t)}{\partial y^{2}}+\frac{\partial^{2} \Phi_{V i}(x, y, z, t)}{\partial z^{2}}\right]+ \\
& +D_{0 \Phi V}\left\{\frac{\partial}{\partial x}\left[g_{\Phi V}(x, y, z, T) \frac{\partial \Phi_{V i-1}(x, y, z, t)}{\partial x}\right]+\frac{\partial}{\partial y}\left[g_{\Phi V}(x, y, z, T) \frac{\partial \Phi_{V i-1}(x, y, z, t)}{\partial y}\right]+\right. \\
& \left.+\frac{\partial}{\partial z}\left[g_{\Phi V}(x, y, z, T) \frac{\partial \Phi_{V i-1}(x, y, z, t)}{\partial z}\right]\right\}, i \geq 1 ;
\end{aligned}
$$

Boundary and initial conditions for the functions takes the form

$$
\begin{aligned}
& \left.\frac{\partial \Phi_{\rho i}(x, y, z, t)}{\partial x}\right|_{x=0}=0,\left.\frac{\partial \Phi_{\rho i}(x, y, z, t)}{\partial x}\right|_{x=L_{x}}=0,\left.\frac{\partial \Phi_{\rho i}(x, y, z, t)}{\partial y}\right|_{y=0}=0,\left.\frac{\partial \Phi_{\rho i}(x, y, z, t)}{\partial y}\right|_{y=L_{y}}=0, \\
& \left.\frac{\partial \Phi_{\rho i}(x, y, z, t)}{\partial z}\right|_{z=0}=0,\left.\frac{\partial \Phi_{\rho i}(x, y, z, t)}{\partial z}\right|_{z=L_{z}}=0, i \geq 0 ; \Phi_{\rho 0}(x, y, z, 0)=f_{\Phi \rho}(x, y, z),
\end{aligned}
$$

$\Phi_{\rho i}(x, y, z, 0)=0, i \geq 1$.

Solutions of the above equations could be written as

$$
\begin{aligned}
& \Phi_{\rho 0}(x, y, z, t)=\frac{1}{L_{x} L_{y} L_{z}}+\frac{2}{L_{x} L_{y} L_{z}} \sum_{n=1}^{\infty} F_{n \Phi_{\rho}} c_{n}(x) c_{n}(y) c_{n}(z) e_{n \Phi_{\rho}}(t)+\frac{2}{L} \sum_{n=1}^{\infty} n c_{n}(x) c_{n}(y) c_{n}(z) \times \\
& \times e_{\Phi_{\rho} n}(t) \int_{0}^{t} e_{\Phi_{\rho^{n}}}(-\tau) \int_{0}^{L_{x}} c_{n}(u) \int_{0}^{L_{y}} c_{n}(v) \int_{0}^{L_{z}} c_{n}(w)\left[k_{I, I}(u, v, w, T) I^{2}(u, v, w, \tau)-\right. \\
& \left.-k_{I}(u, v, w, T) I(u, v, w, \tau)\right] d w d v d u d \tau,
\end{aligned}
$$

where $\quad F_{n \Phi_{\rho}}=\int_{0}^{L_{x}} c_{n}(u) \int_{0}^{L_{y}} c_{n}(v) \int_{0}^{L_{z}} c_{n}(w) f_{\Phi_{\rho}}(u, v, w) d w d v d u, \quad e_{n \Phi_{\rho}}(t)=\exp \left[-\pi^{2} n^{2} D_{0 \Phi_{\rho}} t\left(L_{x}^{-2}+L_{y}^{-2}+L_{z}^{-2}\right)\right]$, $c_{n}(x)=\cos \left(\pi n x / L_{x}\right)$

$$
\begin{aligned}
& \Phi_{\rho i}(x, y, z, t)=-\frac{2 \pi}{L_{x}^{2} L_{y} L_{z}} \sum_{n=1}^{\infty} n c_{n}(x) c_{n}(y) c_{n}(z) e_{\Phi_{\rho} n}(t) \int_{0}^{t} e_{\Phi_{\rho} n}(-\tau) \int_{0}^{L_{x}} s_{n}(u) \int_{0}^{L_{y}} c_{n}(v) \int_{0}^{L_{z}} g_{\Phi_{\rho}}(u, v, w, T) \times \\
& \times c_{n}(w) \frac{\partial \Phi_{I_{\rho} i-1}(u, v, w, \tau)}{\partial u} d w d v d u d \tau-\frac{2 \pi}{L_{x} L_{y}^{2} L_{z}} \sum_{n=1}^{\infty} n c_{n}(x) c_{n}(y) c_{n}(z) e_{\Phi_{\rho} n}(t) \int_{0}^{t} e_{\Phi_{\rho} n}(-\tau) \times \\
& \times \int_{0}^{t} e_{\Phi_{\rho} n}(-\tau) \int_{0}^{L_{x}} c_{n}(u) \int_{0}^{L_{y}} s_{n}(v) \int_{0}^{L_{z}} c_{n}(w) g_{\Phi_{\rho}}(u, v, w, T) \frac{\partial \Phi_{I_{\rho} i-1}(u, v, w, \tau)}{\partial v} d w d v d u d \tau-\frac{2 \pi}{L_{x} L_{y} L_{z}^{2}} \sum_{n=1}^{\infty} n \times \\
& \times e_{\Phi_{\rho} n}(t) \int_{0}^{t} e_{\Phi_{\rho} n}(-\tau) \int_{0}^{L_{x}} c_{n}(u) \int_{0}^{L_{y}} c_{n}(v) \int_{0}^{L_{z}} s_{n}(w) \frac{\partial \Phi_{I_{\rho} i-1}(u, v, w, \tau)}{\partial w} g_{\Phi_{\rho}}(u, v, w, T) d w d v d u d \tau \times
\end{aligned}
$$


On Modelling of Mass Transport for Formulation of Conditions for Increasing Integration Rate of Elements Framework Current Comparator. an Approach to Optimize of Technological Process

$\times c_{n}(x) c_{n}(y) c_{n}(z), i \geq 1$,

where $s_{n}(x)=\sin \left(\pi n x / L_{x}\right)$.

Equations for the functions $C_{i j}(x, y, z, t)(i \geq 0, j \geq 0)$, boundary and initial conditions could be written as $\frac{\partial C_{00}(x, y, z, t)}{\partial t}=D_{0 L} \frac{\partial^{2} C_{00}(x, y, z, t)}{\partial x^{2}}+D_{0 L} \frac{\partial^{2} C_{00}(x, y, z, t)}{\partial y^{2}}+D_{0 L} \frac{\partial^{2} C_{00}(x, y, z, t)}{\partial z^{2}}$;

$\frac{\partial C_{i 0}(x, y, z, t)}{\partial t}=D_{0 L}\left[\frac{\partial^{2} C_{i 0}(x, y, z, t)}{\partial x^{2}}+\frac{\partial^{2} C_{i 0}(x, y, z, t)}{\partial y^{2}}+\frac{\partial^{2} C_{i 0}(x, y, z, t)}{\partial z^{2}}\right]+$

$+D_{0 L} \frac{\partial}{\partial x}\left[g_{L}(x, y, z, T) \frac{\partial C_{i-10}(x, y, z, t)}{\partial x}\right]+D_{0 L} \frac{\partial}{\partial y}\left[g_{L}(x, y, z, T) \frac{\partial C_{i-10}(x, y, z, t)}{\partial y}\right]+$

$+D_{0 L} \frac{\partial}{\partial z}\left[g_{L}(x, y, z, T) \frac{\partial C_{i-10}(x, y, z, t)}{\partial z}\right], i \geq 1$;

$\frac{\partial C_{01}(x, y, z, t)}{\partial t}=D_{0 L} \frac{\partial^{2} C_{01}(x, y, z, t)}{\partial x^{2}}+D_{0 L} \frac{\partial^{2} C_{01}(x, y, z, t)}{\partial y^{2}}+D_{0 L} \frac{\partial^{2} C_{01}(x, y, z, t)}{\partial z^{2}}+$

$+D_{0 L} \frac{\partial}{\partial x}\left[\frac{C_{00}^{\gamma}(x, y, z, t)}{P^{\gamma}(x, y, z, T)} \frac{\partial C_{00}(x, y, z, t)}{\partial x}\right]+D_{0 L} \frac{\partial}{\partial y}\left[\frac{C_{00}^{\gamma}(x, y, z, t)}{P^{\gamma}(x, y, z, T)} \frac{\partial C_{00}(x, y, z, t)}{\partial y}\right]+$

$+D_{0 L} \frac{\partial}{\partial z}\left[\frac{C_{00}^{\gamma}(x, y, z, t)}{P^{\gamma}(x, y, z, T)} \frac{\partial C_{00}(x, y, z, t)}{\partial z}\right]$;

$\frac{\partial C_{02}(x, y, z, t)}{\partial t}=D_{0 L} \frac{\partial^{2} C_{02}(x, y, z, t)}{\partial x^{2}}+D_{0 L} \frac{\partial^{2} C_{02}(x, y, z, t)}{\partial y^{2}}+D_{0 L} \frac{\partial^{2} C_{02}(x, y, z, t)}{\partial z^{2}}+$

$+D_{0 L}\left\{\frac{\partial}{\partial x}\left[C_{01}(x, y, z, t) \frac{C_{00}^{\gamma-1}(x, y, z, t)}{P^{\gamma}(x, y, z, T)} \frac{\partial C_{00}(x, y, z, t)}{\partial x}\right]+\frac{\partial}{\partial y}\left[C_{01}(x, y, z, t) \frac{C_{00}^{\gamma-1}(x, y, z, t)}{P^{\gamma}(x, y, z, T)} \times\right.\right.$

$\left.\left.\times \frac{\partial C_{00}(x, y, z, t)}{\partial y}\right]+\frac{\partial}{\partial z}\left[C_{01}(x, y, z, t) \frac{C_{00}^{\gamma-1}(x, y, z, t)}{P^{\gamma}(x, y, z, T)} \frac{\partial C_{00}(x, y, z, t)}{\partial z}\right]\right\}+$

$\left.\left.\times \frac{\partial C_{00}(x, y, z, t)}{\partial y}\right]+\frac{\partial}{\partial z}\left[C_{01}(x, y, z, t) \frac{C_{00}^{\gamma-1}(x, y, z, t)}{P^{\gamma}(x, y, z, T)} \frac{\partial C_{00}(x, y, z, t)}{\partial z}\right]\right\}+D_{0 L}\left\{\frac{\partial}{\partial x}\left[\frac{C_{00}^{\gamma}(x, y, z, t)}{P^{\gamma}(x, y, z, T)} \times\right.\right.$

$\left.\left.\times \frac{\partial C_{01}(x, y, z, t)}{\partial x}\right]+\frac{\partial}{\partial y}\left[\frac{C_{00}^{\gamma}(x, y, z, t)}{P^{\gamma}(x, y, z, T)} \frac{\partial C_{01}(x, y, z, t)}{\partial y}\right]+\frac{\partial}{\partial z}\left[\frac{C_{00}^{\gamma}(x, y, z, t)}{P^{\gamma}(x, y, z, T)} \frac{\partial C_{01}(x, y, z, t)}{\partial z}\right]\right\}$;

$\frac{\partial C_{11}(x, y, z, t)}{\partial t}=D_{0 L} \frac{\partial^{2} C_{11}(x, y, z, t)}{\partial x^{2}}+D_{0 L} \frac{\partial^{2} C_{11}(x, y, z, t)}{\partial y^{2}}+D_{0 L} \frac{\partial^{2} C_{11}(x, y, z, t)}{\partial z^{2}}+$

$+\left\{\frac{\partial}{\partial x}\left[C_{10}(x, y, z, t) \frac{C_{00}^{\gamma-1}(x, y, z, t)}{P^{\gamma}(x, y, z, T)} \frac{\partial C_{00}(x, y, z, t)}{\partial x}\right]+\frac{\partial}{\partial y}\left[C_{10}(x, y, z, t) \frac{C_{00}^{\gamma-1}(x, y, z, t)}{P^{\gamma}(x, y, z, T)} \times\right.\right.$

$\left.\left.\times \frac{\partial C_{00}(x, y, z, t)}{\partial y}\right]+\frac{\partial}{\partial z}\left[C_{10}(x, y, z, t) \frac{C_{00}^{\gamma-1}(x, y, z, t)}{P^{\gamma}(x, y, z, T)} \frac{\partial C_{00}(x, y, z, t)}{\partial z}\right]\right\} D_{0 L}+$ 
On Modelling of Mass Transport for Formulation of Conditions for Increasing Integration Rate of Elements Framework Current Comparator. an Approach to Optimize of Technological Process

$$
\begin{aligned}
& +D_{0 L}\left\{\frac{\partial}{\partial x}\left[\frac{C_{00}^{\gamma}(x, y, z, t)}{P^{\gamma}(x, y, z, T)} \frac{\partial C_{10}(x, y, z, t)}{\partial x}\right]+\frac{\partial}{\partial y}\left[\frac{C_{00}^{\gamma}(x, y, z, t)}{P^{\gamma}(x, y, z, T)} \frac{\partial C_{10}(x, y, z, t)}{\partial y}\right]+\right. \\
& \left.+\frac{\partial}{\partial z}\left[\frac{C_{00}^{\gamma}(x, y, z, t)}{P^{\gamma}(x, y, z, T)} \frac{\partial C_{10}(x, y, z, t)}{\partial z}\right]\right\}+D_{0 L}\left\{\frac{\partial}{\partial x}\left[g_{L}(x, y, z, T) \frac{\partial C_{01}(x, y, z, t)}{\partial x}\right]+\right. \\
& \left.+\frac{\partial}{\partial y}\left[g_{L}(x, y, z, T) \frac{\partial C_{01}(x, y, z, t)}{\partial y}\right]+\frac{\partial}{\partial z}\left[g_{L}(x, y, z, T) \frac{\partial C_{01}(x, y, z, t)}{\partial z}\right]\right\} ; \\
& \left.\frac{\partial C_{i j}(x, y, z, t)}{\partial x}\right|_{x=0}=0,\left.\frac{\partial C_{i j}(x, y, z, t)}{\partial x}\right|_{x=L_{x}}=0,\left.\frac{\partial C_{i j}(x, y, z, t)}{\partial y}\right|_{y=0}=0,\left.\frac{\partial C_{i j}(x, y, z, t)}{\partial y}\right|_{y=L_{y}}=0, \\
& \left.\frac{\partial C_{i j}(x, y, z, t)}{\partial z}\right|_{z=0}=0,\left.\frac{\partial C_{i j}(x, y, z, t)}{\partial z}\right|_{z=L_{z}}=0, i \geq 0, j \geq 0 ;
\end{aligned}
$$

$C_{00}(x, y, z, 0)=f_{C}(x, y, z), C_{i j}(x, y, z, 0)=0, i \geq 1, j \geq 1$.

Functions $C_{i j}(x, y, z, t)(i \geq 0, j \geq 0)$ could be approximated by the following series during solutions of the above equations

$$
C_{00}(x, y, z, t)=\frac{F_{0 C}}{L_{x} L_{y} L_{z}}+\frac{2}{L_{x} L_{y} L_{z}} \sum_{n=1}^{\infty} F_{n C} c_{n}(x) c_{n}(y) c_{n}(z) e_{n C}(t) .
$$

Here $e_{n C}(t)=\exp \left[-\pi^{2} n^{2} D_{0 C} t\left(\frac{1}{L_{x}^{2}}+\frac{1}{L_{y}^{2}}+\frac{1}{L_{z}^{2}}\right)\right], F_{n C}=\int_{0}^{L_{x}} c_{n}(u) \int_{0}^{L_{y}} c_{n}(v) \int_{0}^{L_{z}} f_{C}(u, v, w) c_{n}(w) d w d v d u$;

$$
C_{i 0}(x, y, z, t)=-\frac{2 \pi}{L_{x}^{2} L_{y} L_{z}} \sum_{n=1}^{\infty} n F_{n C} c_{n}(x) c_{n}(y) c_{n}(z) e_{n C}(t) \int_{0}^{t} e_{n C}(-\tau) \int_{0}^{L_{x}} s_{n}(u) \int_{0}^{L_{y}} c_{n}(v) \int_{0}^{L_{z}} g_{L}(u, v, w, T) \times
$$$$
\times c_{n}(w) \frac{\partial C_{i-10}(u, v, w, \tau)}{\partial u} d w d v d u d \tau-\frac{2 \pi}{L_{x} L_{y}^{2} L_{z}} \sum_{n=1}^{\infty} n F_{n C} c_{n}(x) c_{n}(y) c_{n}(z) e_{n C}(t) \int_{0}^{t} e_{n C}(-\tau) \times
$$$$
\times \int_{0}^{L_{x}} c_{n}(u) \int_{0}^{L_{y}} s_{n}(v) \int_{0}^{L_{z}} c_{n}(v) g_{L}(u, v, w, T) \frac{\partial C_{i-10}(u, v, w, \tau)}{\partial v} d w d v d u d \tau-\frac{2 \pi}{L_{x} L_{y} L_{z}^{2}} \sum_{n=1}^{\infty} n F_{n C} e_{n C}(t) \times
$$$$
\times c_{n}(x) c_{n}(y) c_{n}(z) \int_{0}^{t} e_{n C}(-\tau) \int_{0}^{L_{x}} c_{n}(u) \int_{0}^{L_{y}} c_{n}(v) \int_{0}^{L_{z}} s_{n}(v) g_{L}(u, v, w, T) \frac{\partial C_{i-10}(u, v, w, \tau)}{\partial w} d w d v d u d \tau, i \geq 1
$$$$
C_{01}(x, y, z, t)=-\frac{2 \pi}{L_{x}^{2} L_{y} L_{z}} \sum_{n=1}^{\infty} n F_{n C} c_{n}(x) c_{n}(y) c_{n}(z) e_{n C}(t) \int_{0}^{t} e_{n C}(-\tau) \int_{0}^{L_{x}} s_{n}(u) \int_{0}^{L_{y}} c_{n}(v) \int_{0}^{L_{z}} c_{n}(w) \times
$$$$
\times \frac{C_{00}^{\gamma}(u, v, w, \tau)}{P^{\gamma}(u, v, w, T)} \frac{\partial C_{00}(u, v, w, \tau)}{\partial u} d w d v d u d \tau-\frac{2 \pi}{L_{x} L_{y}^{2} L_{z}} \sum_{n=1}^{\infty} n F_{n C} c_{n}(x) c_{n}(y) c_{n}(z) e_{n C}(t) \times
$$$$
\times \int_{0}^{t} e_{n C}(-\tau) \int_{0}^{L_{x}} c_{n}(u) \int_{0}^{L_{y}} s_{n}(v) \int_{0}^{L_{z}} c_{n}(w) \frac{C_{00}^{\gamma}(u, v, w, \tau)}{P^{\gamma}(u, v, w, T)} \frac{\partial C_{00}(u, v, w, \tau)}{\partial v} d w d v d u d \tau-\frac{2 \pi}{L_{x} L_{y} L_{z}^{2}} \sum_{n=1}^{\infty} n e_{n C}(t) \times
$$

$\times F_{n C} c_{n}(x) c_{n}(y) c_{n}(z) \int_{0}^{t} e_{n C}(-\tau) \int_{0}^{L_{x}} c_{n}(u) \int_{0}^{L_{y}} c_{n}(v) \int_{0}^{L_{z}} s_{n}(w) \frac{C_{00}^{\gamma}(u, v, w, \tau)}{P^{\gamma}(u, v, w, T)} \frac{\partial C_{00}(u, v, w, \tau)}{\partial w} d w d v d u d \tau$; 
On Modelling of Mass Transport for Formulation of Conditions for Increasing Integration Rate of Elements Framework Current Comparator. an Approach to Optimize of Technological Process

$C_{02}(x, y, z, t)=-\frac{2 \pi}{L_{x}^{2} L_{y} L_{z}} \sum_{n=1}^{\infty} n F_{n C} c_{n}(x) c_{n}(y) c_{n}(z) e_{n C}(t) \int_{0}^{t} e_{n C}(-\tau) \int_{0}^{L_{x}} s_{n}(u) \int_{0}^{L_{y}} c_{n}(v) \int_{0}^{L_{z}} c_{n}(w) \times$ $\times C_{01}(u, v, w, \tau) \frac{C_{00}^{\gamma-1}(u, v, w, \tau)}{P^{\gamma}(u, v, w, T)} \frac{\partial C_{00}(u, v, w, \tau)}{\partial u} d w d v d u d \tau-\frac{2 \pi}{L_{x} L_{y}^{2} L_{z}} \sum_{n=1}^{\infty} F_{n C} c_{n}(x) c_{n}(y) \times$ $\times n c_{n}(z) e_{n C}(t) \int_{0}^{t} e_{n C}(-\tau) \int_{0}^{L_{x}} c_{n}(u) \int_{0}^{L_{y}} s_{n}(v) \int_{0}^{L_{z}} C_{01}(u, v, w, \tau) \frac{C_{00}^{\gamma-1}(u, v, w, \tau)}{P^{\gamma}(u, v, w, T)} \frac{\partial C_{00}(u, v, w, \tau)}{\partial v} \times$ $\times c_{n}(w) d w d v d u d \tau-\frac{2 \pi}{L_{x} L_{y} L_{z}^{2}} \sum_{n=1}^{\infty} n F_{n C} c_{n}(x) c_{n}(y) c_{n}(z) e_{n C}(t) \int_{0}^{t} e_{n C}(-\tau) \int_{0}^{L_{x}} c_{n}(u) \int_{0}^{L_{y}} c_{n}(v) \times$ $\times \int_{0}^{L_{z}} s_{n}(w) C_{01}(u, v, w, \tau) \frac{C_{00}^{\gamma-1}(u, v, w, \tau)}{P^{\gamma}(u, v, w, T)} \frac{\partial C_{00}(u, v, w, \tau)}{\partial w} d w d v d u d \tau-\frac{2 \pi}{L_{x}^{2} L_{y} L_{z}} \sum_{n=1}^{\infty} n c_{n}(x) \times$ $\times F_{n C} c_{n}(y) c_{n}(z) e_{n C}(t) \int_{0}^{t} e_{n C}(-\tau) \int_{0}^{L_{x}} s_{n}(u) \int_{0}^{L_{y}} c_{n}(v) \int_{0}^{L_{z}} c_{n}(w) C_{01}(u, v, w, \tau) \frac{\partial C_{00}(u, v, w, \tau)}{\partial u} \times$ $\times \frac{C_{00}^{\gamma-1}(u, v, w, \tau)}{P^{\gamma}(u, v, w, T)} d w d v d u d \tau-\frac{2 \pi}{L_{x} L_{y}^{2} L_{z}} \sum_{n=1}^{\infty} n F_{n C} c_{n}(x) c_{n}(y) c_{n}(z) e_{n C}(t) \int_{0}^{t} e_{n C}(-\tau) \int_{0}^{L_{x}} c_{n}(u) \times$ $\times \int_{0}^{L_{y}} s_{n}(v) \int_{0}^{L_{z}} c_{n}(w) C_{01}(u, v, w, \tau) \frac{C_{00}^{\gamma-1}(u, v, w, \tau)}{P^{\gamma}(u, v, w, T)} \frac{\partial C_{00}(u, v, w, \tau)}{\partial v} d w d v d u d \tau-\frac{2 \pi}{L_{x} L_{y} L_{z}^{2}} \sum_{n=1}^{\infty} n \times$ $\times F_{n C} c_{n}(x) c_{n}(y) c_{n}(z) e_{n C}(t) \int_{0}^{t} e_{n C}(-\tau) \int_{0}^{L_{x}} c_{n}(u) \int_{0}^{L_{y}} c_{n}(v) \int_{0}^{L_{z}} s_{n}(w) C_{01}(u, v, w, \tau) \frac{C_{00}^{\gamma-1}(u, v, w, \tau)}{P^{\gamma}(u, v, w, T)} \times$ $\times \frac{\partial C_{00}(u, v, w, \tau)}{\partial w} d w d v d u d \tau-\frac{2 \pi}{L_{x}^{2} L_{y} L_{z}} \sum_{n=1}^{\infty} F_{n C} c_{n}(x) c_{n}(y) c_{n}(z) e_{n C}(t) \int_{0}^{t} e_{n C}(-\tau) \int_{0}^{L_{x}} s_{n}(u) \times$ $\times n \int_{0}^{L_{y}} c_{n}(v) \int_{0}^{L_{z}} c_{n}(w) \frac{C_{00}^{\gamma}(u, v, w, \tau)}{P^{\gamma}(u, v, w, T)} \frac{\partial C_{01}(u, v, w, \tau)}{\partial u} d w d v d u d \tau-\frac{2 \pi}{L_{x} L_{y}^{2} L_{z}} \sum_{n=1}^{\infty} c_{n}(x) e_{n C}(t) \times$ $\times F_{n C} c_{n}(y) \int_{0}^{t} e_{n C}(-\tau) \int_{0}^{L_{x}} c_{n}(u) \int_{0}^{L_{y}} s_{n}(v) \int_{0}^{L_{z}} c_{n}(w) \frac{C_{00}^{\gamma}(u, v, w, \tau)}{P^{\gamma}(u, v, w, T)} \frac{\partial C_{01}(u, v, w, \tau)}{\partial v} d w d v d u d \tau \times$ $\times n c_{n}(z)-\frac{2 \pi}{L_{x} L_{y} L_{z}^{2}} \sum_{n=1}^{\infty} n F_{n C} c_{n}(x) c_{n}(y) c_{n}(z) e_{n C}(t) \int_{0}^{t} e_{n C}(-\tau) \int_{0}^{L_{x}} c_{n}(u) \int_{0}^{L_{y}} c_{n}(v) \int_{0}^{L_{z}} s_{n}(w) \times$ $\times \frac{C_{00}^{\gamma}(u, v, w, \tau)}{P^{\gamma}(u, v, w, T)} \frac{\partial C_{01}(u, v, w, \tau)}{\partial w} d w d v d u d \tau$

$C_{11}(x, y, z, t)=-\frac{2 \pi}{L_{x}^{2} L_{y} L_{z}} \sum_{n=1}^{\infty} n F_{n C} c_{n}(x) c_{n}(y) c_{n}(z) e_{n C}(t) \int_{0}^{t} e_{n C}(-\tau) \int_{0}^{L_{x}} s_{n}(u) \int_{0}^{L_{y}} c_{n}(v) \int_{0}^{L_{z}} c_{n}(w) \times$ $\times g_{L}(u, v, w, T) \frac{\partial C_{01}(u, v, w, \tau)}{\partial u} d w d v d u d \tau-\frac{2 \pi}{L_{x} L_{y}^{2} L_{z}} \sum_{n=1}^{\infty} n F_{n C} c_{n}(x) c_{n}(y) c_{n}(z) e_{n C}(t) \times$ 
On Modelling of Mass Transport for Formulation of Conditions for Increasing Integration Rate of Elements Framework Current Comparator. an Approach to Optimize of Technological Process

$$
\begin{aligned}
& \times \int_{0}^{t} e_{n C}(-\tau) \int_{0}^{L_{x}} c_{n}(u) \int_{0}^{L_{y}} s_{n}(v) \int_{0}^{L_{z}} c_{n}(w) g_{L}(u, v, w, T) \frac{\partial C_{01}(u, v, w, \tau)}{\partial v} d w d v d u d \tau-\frac{2 \pi}{L_{x} L_{y} L_{z}^{2}} \times \\
& \times \sum_{n=1}^{\infty} n e_{n C}(t) \int_{0}^{t} e_{n C}(-\tau) \int_{0}^{L_{x}} c_{n}(u) \int_{0}^{L_{y}} c_{n}(v) \int_{0}^{L_{z}} s_{n}(w) g_{L}(u, v, w, T) \frac{\partial C_{01}(u, v, w, \tau)}{\partial w} d w d v d u d \tau \times \\
& \times F_{n C} c_{n}(x) c_{n}(y) c_{n}(z)-\frac{2 \pi}{L_{x}^{2} L_{y} L_{z}} \sum_{n=1}^{\infty} F_{n C} c_{n}(x) c_{n}(y) c_{n}(z) e_{n C}(t) \int_{0}^{t} e_{n C}(-\tau) \int_{0}^{L_{x}} s_{n}(u) \int_{0}^{L_{y}} c_{n}(v) \times \\
& \times n \int_{0}^{L_{z}} c_{n}(w) \frac{C_{00}^{\gamma}(u, v, w, \tau)}{P^{\gamma}(u, v, w, T)} \frac{\partial C_{10}(u, v, w, \tau)}{\partial u} d w d v d u d \tau-\frac{2 \pi}{L_{x} L_{y}^{2} L_{z}} \sum_{n=1}^{\infty} n F_{n C} c_{n}(x) c_{n}(y) \times \\
& \times c_{n}(z) e_{n C}(t) \int_{0}^{t} e_{n C}(-\tau) \int_{0}^{L_{x}} c_{n}(u) \int_{0}^{L_{y}} s_{n}(v) \int_{0}^{L_{z}} c_{n}(w) \frac{C_{00}^{\gamma}(u, v, w, \tau)}{P^{\gamma}(u, v, w, T)} \frac{\partial C_{10}(u, v, w, \tau)}{\partial v} d w d v d u d \tau- \\
& -\frac{2 \pi}{L_{x} L_{y} L_{z}^{2}} \sum_{n=1}^{\infty} n F_{n C} c_{n}(x) c_{n}(y) c_{n}(z) e_{n C}(t) \int_{0}^{t} e_{n C}(-\tau) \int_{0}^{L_{x}} c_{n}(u) \int_{0}^{L_{y}} c_{n}(v) \int_{0}^{L_{z}} s_{n}(w) \frac{C_{00}^{\gamma}(u, v, w, \tau)}{P^{\gamma}(u, v, w, T)} \times \\
& \times \frac{\partial C_{10}(u, v, w, \tau)}{\partial w} d w d v d u d \tau-\frac{2 \pi}{L_{x}^{2} L_{y} L_{z}} \sum_{n=1}^{\infty} n F_{n C} c_{n}(x) c_{n}(y) c_{n}(z) e_{n C}(t) \int_{0}^{t} e_{n C}(-\tau) \int_{0}^{L_{x}} s_{n}(u) \times \\
& \times \int_{0}^{L_{y}} c_{n}(v) \int_{0}^{L_{z}} c_{n}(w) C_{10}(u, v, w, \tau) \frac{C_{00}^{\gamma-1}(u, v, w, \tau)}{P^{\gamma}(u, v, w, T)} \frac{\partial C_{00}(u, v, w, \tau)}{\partial u} d w d v d u d \tau-\frac{2 \pi}{L_{x} L_{y}^{2} L_{z}} \sum_{n=1}^{\infty} n \times \\
& \times F_{n C} c_{n}(x) c_{n}(y) c_{n}(z) e_{n C}(t) \int_{0}^{t} e_{n C}(-\tau) \int_{0}^{L_{x}} c_{n}(u) \int_{0}^{L_{y}} s_{n}(v) \int_{0}^{L_{z}} c_{n}(w) \frac{C_{00}^{\gamma-1}(u, v, w, \tau)}{P^{\gamma}(u, v, w, T)} \frac{\partial C_{00}(u, v, w, \tau)}{\partial v} \times \\
& \times C_{10}(u, v, w, \tau) d w d v d u d \tau-\frac{2 \pi}{L_{x} L_{y} L_{z}^{2}} \sum_{n=1}^{\infty} n F_{n C} c_{n}(x) c_{n}(y) c_{n}(z) e_{n C}(t) \int_{0}^{t} e_{n C}(-\tau) \int_{0}^{L_{x}} c_{n}(u) \times \\
& \times \int_{0}^{L_{y}} c_{n}(v) \int_{0}^{L_{z}} s_{n}(w) C_{10}(u, v, w, \tau) \frac{C_{00}^{\gamma-1}(u, v, w, \tau)}{P^{\gamma}(u, v, w, T)} \frac{\partial C_{00}(u, v, w, \tau)}{\partial w} d w d v d u d \tau .
\end{aligned}
$$

Citation: E.L. Pankratov (2021). “ On Modelling of Mass Transport for Formulation of Conditions for Increasing Integration Rate of Elements Framework Current Comparator. an Approach to Optimize of Technological Process." International Journal of Innovative Research in Electronics and Communications (IJIREC), 8(1), pp.9-28. http://dx.Doi.org/10. 20431/2349-4050.0801002

Copyright: () 2021 Authors. This is an open-access article distributed under the terms of the Creative Commons Attribution License, which permits unrestricted use, distribution, and reproduction in any medium, provided the original author and source are credited. 